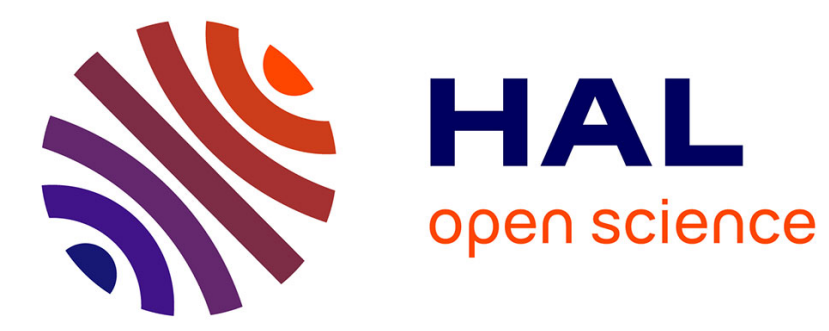

\title{
Hadamard well-posedness for a class of nonlinear shallow shell problems
}

John Cagnol, Irena Lasiecka, Catherine Lebiedzik, Richard Marchand

\section{To cite this version:}

John Cagnol, Irena Lasiecka, Catherine Lebiedzik, Richard Marchand. Hadamard well-posedness for a class of nonlinear shallow shell problems. Nonlinear Analysis: Theory, Methods and Applications, 2007, 67 (8), pp.2452-2484. 10.1016/j.na.2006.09.004 . hal-01570319

\section{HAL Id: hal-01570319 https://hal.science/hal-01570319}

Submitted on 26 Aug 2017

HAL is a multi-disciplinary open access archive for the deposit and dissemination of scientific research documents, whether they are published or not. The documents may come from teaching and research institutions in France or abroad, or from public or private research centers.
L'archive ouverte pluridisciplinaire HAL, est destinée au dépôt et à la diffusion de documents scientifiques de niveau recherche, publiés ou non, émanant des établissements d'enseignement et de recherche français ou étrangers, des laboratoires publics ou privés. 


\title{
Hadamard well-posedness for a class of nonlinear shallow shell problems
}

\author{
John Cagnol $^{\mathrm{a}, *}$, Irena Lasiecka ${ }^{\mathrm{b}}$, Catherine Lebiedzik $^{\mathrm{c}}$, Richard Marchand ${ }^{\mathrm{d}}$ \\ a Pôle Universitaire Léonard de Vinci, ESILV, DER CS, 92916 Paris La Défense, France \\ ${ }^{\mathrm{b}}$ University of Virginia, Department of Mathematics, Charlottesville, VA 22904, USA \\ ${ }^{\mathrm{c}}$ Wayne State University, Department of Mathematics, Detroit, MI 48201, USA \\ d Slippery Rock University, Department of Mathematics, Slippery Rock, PA 16057, USA
}

Received 23 December 2005; accepted 7 September 2006

\begin{abstract}
This paper is concerned with the nonlinear shallow shell model introduced in 1966 by W.T. Koiter in [On the nonlinear theory of thin elastic shells. III, Nederl. Akad. Wetensch. Proc. Ser. B 69 (1966) 33-54, Section 11] and later studied in [M. Bernadou, J.T. Oden, An existence theorem for a class of nonlinear shallow shell problems, J. Math. Pures Appl. (9) 60(3) (1981) 285-308]. We consider a version of this model which is based upon the intrinsic shell modeling techniques introduced by Michel Delfour and Jean-Paul Zolésio. We show existence and uniqueness of both regular and weak solutions to the dynamical model and that the solutions are continuous with respect to the initial data. While existence and uniqueness of regular solutions to nonlinear dynamic shell equations has been known, full Hadamard well-posedness of weak solutions, as shown in this paper, is a new result which solves an old open problem in the field.
\end{abstract}

(C) 2006 Elsevier Ltd. All rights reserved.

MSC: 35Q99; 35L75; 74K25

Keywords: Uniqueness; Continuous dependence; Hyperbolic partial differential equation; Koiter nonlinear shell model; Intrinsic geometric shell modeling

\section{Introduction}

In [21], Koiter classifies shell problems according to the order of magnitude of the deflection. He arranges the classes in an order of increasing specialization, as follows: Large Deflections, characterized by the absence of restriction as to the magnitude of the displacement; Moderate Deflections, characterized by small values of all displacement gradients with respect to unity, although nothing is assumed a priori about their relative orders of magnitude; Small Finite Deflections, characterized by small displacement gradients and by rotations whose squares do not exceed the middle surface strain in order of magnitude; Infinitesimal Deflections, characterized by small displacement gradients none of which exceed the middle surface strains in order of magnitude.

\footnotetext{
* Corresponding author. Tel.: +331411671 88; fax: +33141167171.

E-mail address: john.cagnol@devinci.fr (J. Cagnol).
} 
The infinitesimal deflections hypothesis leads to the well-known linear theory. Instead, in this article we shall consider the small finite deflections case, which is studied in [21, Section 11]. This corresponds to the "medium bending case" in the sense of Naghdi (see [28]) and to the "approximation of small strains and moderately small rotations" in the sense of Lyell and Sanders (see [29]). This case leads to the geometrically nonlinear model discussed in [3] and summarized in [2, Section 7.3]. In [3], Bernadou and Oden have proven that a solution to the static model exists and that it is unique provided the load is sufficiently small. Philippe G. Ciarlet proposed in [8] a model where the exact change of curvature tensor is modified and Liliana Gratie generalized this latter model to the case of nonconstant thickness in [17]. See [10] for the linear and nonlinear theories of shells.

This paper is concerned with the dynamical model. Dynamical systems of nonlinear elasticity have attracted much interest over the years $[32,9,22,14,18,13]$. Numerous physical phenomena can be described by these models, which mathematically take the form of a set of coupled partial differential equations. The shell model considered here is a nonlinear, strongly coupled system of hyperbolic equations with clamped (Dirichlet) boundary conditions. Its counterpart for a flat geometry is the full von Kármán system [32,9], which couples a plate-like equation for the normal displacement variable with wave equations for the tangential (in-plane) displacements.

Existence of weak solutions for such models follows from standard Faedo-Galerkin method [27]. However, the issue of well-posedness (uniqueness and continuous dependence on initial data) is much more subtle. The nonlinear term in the equation is neither locally Lipschitz nor bounded in the finite energy space. In addition, the problem is not monotone and weak solutions do not exhibit any additional regularity properties (unlike parabolic-like problems). Thus typical or known methods used for proving well-posedness of weak solutions are no longer applicable.

It is the main goal set in this paper to provide an affirmative answer to full Hadamard well-posedness of weak (often referred to as finite energy) solutions. More specifically, the main contribution of this work is threefold:

- Existence and uniqueness of weak solutions (i.e. finite energy solutions).

- Continuous dependence of solutions with respect to initial data measured in finite energy norm.

- Regularity of weak solutions.

We shall use the intrinsic geometry method of Michel Delfour and Jean-Paul Zolésio, which relies on the oriented distance function to describe the geometry [12,11]. This technique takes advantage of the intrinsic geometric properties of the shell. Here, the shell is described in terms of tangential differential operators which are defined by means of the oriented boundary distance function in $\mathbb{R}^{3}$. Sobolev spaces, Green's formula, and key inequalities such as Poincaré and Korn's inequalities are all well defined. Delfour and Zolésio have constructed models under a variety of assumptions. A linear version of the model developed in this paper was introduced in $[5,6]$.

This model is a geometrically expanded version of the full (vectorial) von Kármán system, which has been rather extensively studied. Much literature however deals with a static or semi-static model for which the mathematical issues are quite different (both in the case of the plate [9] and in that of the shell [3]). In the dynamic case, there are many results available for the modified (scalar) von Kármán model, which does not account for the in-plane displacements. This modification results in a scalar model where the nonlinearity appears non-locally via the Airy stress function. The scalar von Kármán model has been extensively studied in the literature with many results on well-posedness and stability available [7,4,15,19,22]. It is worth saying that much of the rich theory developed for scalar von Kármán evolutions owes its success to the rather unexpected regularity of the Airy stress function [15]. In the full (vectorial) von Kármán system the Airy stress function plays no role. Thus, the benefits of additional regularity of the nonlinear term are not available. However, the simplification of ignoring the in-plane displacements does not make sense in the case of a shell. Thus, in the case of nonlinear shells the relevant dynamic model is that of vectorial structure. The full vectorial system is much more complicated than the modified version, due to the strong coupling between hyperbolic equations. The real difficulty lies in the lack of regularity of the nonlinear terms in the finite energy norms, at least in the two-dimensional case (the one-dimensional case was treated in [23]). The twodimensional case with homogeneous Dirichlet boundary data was considered in [30] where uniqueness of both regular and finite energy solutions was derived. A related result is [31] which relies on Strichartz-type estimates and covers the cases of the whole plane and of special geometries such as hinged rectangular plates. A full well-posedness theory for these equations was finally provided in [26] which proves existence and uniqueness of weak, intermediate and regular solutions under a variety of boundary conditions including free boundary conditions with nonlinear boundary dissipation. This proof is based in part on the method introduced by Sedenko [30] for the Marguerre-Vlasov equations. 
In addition, Hadamard well-posedness is proved in [18] by application of a finite difference method which allows for the rigorous derivation of an appropriate energy identity for the system.

It is the aim of this work to further extend the techniques developed in the case of "flat" geometry [26,18] to the curved case required by a shell model. The final result is full Hadamard well-posedness of finite energy solutions governing nonlinear dynamic shells.

Throughout this paper we will denote by $\mathcal{S}_{l}$ the shell and by $\Gamma$ its mid-surface. We shall make the following hypothesis:

(i) The shell is assumed to be made of an isotropic and homogeneous material, so that the Lamé coefficients $\lambda>0$ and $\mu>0$ are constant. The density of the material will be denoted as $\rho>0$.

(ii) (shallowness assumption) The thickness $l$ of the shell is small enough to accommodate the curvatures $H$ and $K$, i.e. the product of the thickness and the curvatures is small as compared to 1 . In addition, the shell is shallow in the sense that the second fundamental form (here given in terms of the oriented distance function as $D^{2} b$ ) and its derivative $\left(D^{3} b\right)$ are small. This assumption allows us to neglect certain terms in the strain energy in comparison to the model in [5,6] and yields a model in which the coupling between the normal and tangential displacements is of the first (rather than third) order. For a detailed justification of these assumptions we refer the reader to Koiter [20,21].

(iii) (Kirchhoff hypothesis) In the classical thin plate theory named after Kirchhoff, the displacement vectors of the shell $\mathcal{S}_{l}$ and of the mid-surface $\Gamma$ are related by the hypothesis that the filaments of the plate initially perpendicular to the middle surface remain straight and perpendicular to the deformed surface, and undergo neither contraction nor extension. We generalize this hypothesis to the case of a shell using the intrinsic geometry in [5].

For the sake of self-containment, we provide background material on intrinsic modeling in Section 2. In Section 3.3, we state the main findings of this paper. Section 5 is concerned with establishing preliminary results that will be used in the subsequent sections. Section 4 is concerned with describing the model [2, Section 7.3] and subsequently justifying the investigation of the equations presented in (12). We prove the existence result in Section 6, the uniqueness result in Section 7, and the continuous dependence with respect to the initial data in Section 8.

\section{Intrinsic modeling}

In this section we present a brief overview of the oriented distance function and the intrinsic tangential calculus that forms the basis of our shell model.

\subsection{The oriented distance function and the intrinsic geometry}

In order to improve readability we here include a brief discussion of the oriented distance function and the intrinsic geometric methods of Delfour and Zolésio. Since by necessity this overview will lack detail, the reader is referred to $[12,11]$ for a definitive exposition on this topic.

Consider a domain $\mathcal{O} \subset \mathbb{R}^{3}$ whose non-empty boundary $\partial \mathcal{O}$ is a $C^{1}$ two-dimensional submanifold of $\mathbb{R}^{3}$. Define the oriented (or signed) distance function to $\mathcal{O}$ as

$$
b(x)=d_{\mathcal{O}}(x)-d_{\mathbb{R}^{3} \backslash \mathcal{O}}(x)
$$

where $d$ is the Euclidean distance from the point $x$ to the domain $\mathcal{O}$. In other words, $b(x)$ is simply the positive or negative distance to the boundary $\partial \mathcal{O}$, depending on whether we are outside or inside the domain $\mathcal{O}$. It can be shown that for every $x \in \partial \mathcal{O}$, there exists a neighborhood where the function $\nabla b=v$, the unit outward external normal to $\partial \mathcal{O}[11]$.

Consider a subset $\Gamma \subseteq \partial \mathcal{O}$ which will eventually become the mid-surface of our shell. We define the projection $p(x)$ of a point $x$ onto $\Gamma$ as $p(x)=x-b(x) \nabla b(x)$. Then, we define a shell $\mathcal{S}_{l}$ of thickness $l$ as (see Fig. 1)

$$
\mathcal{S}_{l}(\Gamma) \equiv\left\{x \in \mathbb{R}^{3}: p(x) \in \Gamma,|b(x)|<l / 2\right\} .
$$

A natural curvilinear coordinate system $(X, z)$ is thus induced on the shell $\mathcal{S}_{l}$, where the coordinate vector $X$ gives the position of a point on the mid-surface $\Gamma$, and $z \in\left(-\frac{l}{2}, \frac{l}{2}\right)$ gives the vertical (normal) distance from the mid-surface. 


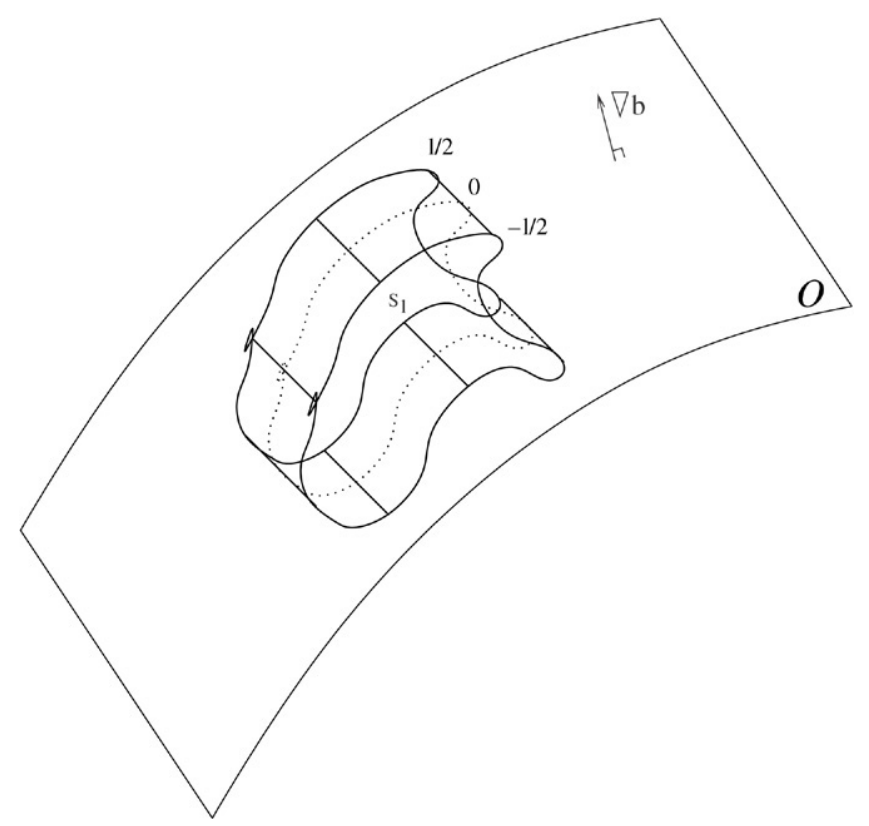

Fig. 1. The shell.

Using this notation, we also define the "flow mapping" $T_{z}(X)$ as

$$
T_{z}(X)=X+z \nabla b(X)
$$

for all $X$ and $z$ in $\mathcal{S}_{l}$. This allows us to reconstruct the action at a given height $z$ of the shell, once we know the action of the mid-surface $\Gamma$. Define as $\Gamma^{z}$ the surface $T_{z}(\Gamma)$ at the 'altitude' $z$. Then, one can also describe the shell $\mathcal{S}_{l}$ as

$$
\mathcal{S}_{l}=\bigcup_{z=-l / 2}^{l / 2} \Gamma_{z} .
$$

The curvatures of the shell will be denoted as $H$ and $K$. These can be reconstructed from the boundary distance function $b(x)$ by noting that at any point $(X, z)$, the matrix $D^{2} b$ has eigenvalues $0, \lambda_{1}, \lambda_{2}$. The curvatures are then given by $\operatorname{tr}\left(D^{2} b\right)=2 H=\lambda_{1}+\lambda_{2}$ and $K=\lambda_{1} \lambda_{2}$.

\subsection{Tangential differential calculus}

Next, we mention briefly some useful aspects of the tangential differential calculus. Given $f \in C^{1}(\Gamma)$, we define the tangential gradient $\nabla_{\Gamma}$ of the scalar function $f$ by means of the projection as

$$
\left.\nabla_{\Gamma} f \equiv \nabla(f \circ p)(x)\right|_{\Gamma} .
$$

This notion of the tangential gradient is equivalent to the classical definition using an extension $F$ of $f$ in the neighborhood of $\Gamma$, i.e. $\nabla_{\Gamma} f=\left.\nabla F\right|_{\Gamma}-\frac{\partial F}{\partial v} v$ [11]. Following the same idea we can define the tangential Jacobian matrix of a vector function $v \in C^{1}(\Gamma)^{3}$ as

$$
\left.D_{\Gamma} v \equiv D(v \circ p)\right|_{\Gamma} \quad \text { or } \quad\left(D_{\Gamma} v\right)_{i j}=\left(\nabla_{\Gamma} v_{i}\right)_{j},
$$

the tangential divergence as

$$
\left.\operatorname{div}_{\Gamma} v \equiv \operatorname{div}(v \circ p)\right|_{\Gamma},
$$

the Hessian $D_{\Gamma}^{2} f$ of $f \in C^{2}(\Gamma)$ as

$$
D_{\Gamma}^{2} f=D_{\Gamma}\left(\nabla_{\Gamma} f\right),
$$


the Laplace-Beltrami operator of $f \in C^{2}(\Gamma)$ as

$$
\Delta_{\Gamma} f \equiv \operatorname{div}_{\Gamma}\left(\nabla_{\Gamma} f\right)=\left.\Delta(f \circ p)\right|_{\Gamma},
$$

the tangential linear strain tensor of elasticity as

$$
\varepsilon_{\Gamma}(v) \equiv \frac{1}{2}\left(D_{\Gamma} v+{ }^{*} D_{\Gamma} v\right)=\left.\varepsilon(v \circ p)\right|_{\Gamma}
$$

and the tangential vectorial divergence of a second-order tensor $A$ as

$$
\left.\operatorname{div}_{\Gamma} A \equiv \operatorname{div}(A \circ p)\right|_{\Gamma}=\operatorname{div}_{\Gamma} A_{i} .
$$

Using the definitions given in (4) one can derive Green's formula in the tangential calculus [11]:

$$
\int_{\Gamma} f \operatorname{div}_{\Gamma} v \mathrm{~d} \Gamma+\int_{\Gamma}\left\langle\nabla_{\Gamma} f, v\right\rangle \mathrm{d} \Gamma=\int_{\Upsilon}\langle f v, v\rangle \mathrm{d} \Upsilon+2 \int_{\Gamma} f H\langle v, \nabla b\rangle \mathrm{d} \Gamma
$$

where $v$ is the outward unit normal to the curve $\Upsilon$. We use the definition of $\varepsilon_{\Gamma}(u)$ and of the tangential vectorial divergence of a second-order tensor $A$ as

$$
\left.\operatorname{div}_{\Gamma} A \equiv \operatorname{div}(A \circ p)\right|_{\Gamma}=\operatorname{div}_{\Gamma} A_{i}
$$

to derive the integration formula

$$
\int_{\Gamma} \operatorname{tr}\left(\varepsilon_{\Gamma}(u) A\right) \mathrm{d} \Gamma=\int_{\partial \Gamma}\langle u, A v\rangle \mathrm{d}(\partial \Gamma)-\int_{\Gamma}\left\langle u, \operatorname{div}_{\Gamma} A\right\rangle \mathrm{d} \Gamma+\int_{\Gamma} 2 H\langle u, A \nabla b\rangle \mathrm{d} \Gamma
$$

for $A$ a symmetric matrix. Finally, from $[11,12]$ we have that

$$
\left\langle\nabla_{\Gamma} w, \nabla b\right\rangle=0, \quad D_{\Gamma} v \nabla b=0
$$

by definition for any scalar $w$ and vector $v$. In addition, if we consider a purely tangent vector $v=v_{\Gamma}$, i.e. $\left\langle v_{\Gamma}, \nabla b\right\rangle=0$, we can take the tangential gradient of both sides of this expression and derive the following useful formula

$$
D^{2} b v_{\Gamma}+{ }^{*} D_{\Gamma} v_{\Gamma} \nabla b=0 .
$$

In the case of tangential $v_{\Gamma}$, the last term of Eq. (5) is also zero. We conclude this section with some remarks about notation. We shall adopt the following notation:

$$
|w|_{s, \Gamma} \equiv|w|_{H^{s}(\Gamma)} ; \quad(u, v)_{\Gamma} \equiv \int_{\Gamma} u v \mathrm{~d} \Gamma
$$

and use $\langle\cdot, \cdot\rangle$ to denote the scalar product of two vectors. Throughout this paper the conventions of [16] concerning tensors are used. For instance, we will make no distinction between a second-order tensor and a matrix, nor will we make a distinction between a first-order tensor and a vector. Consequently we will not distinguish simple contraction and multiplication. Finally, the notation $\partial_{t} \varphi$ is used to denote the partial derivative of $\varphi$ with respect to the time variable, and the notation $A \cdots B$ denotes the double contraction of two matrices - i.e. $A \cdots B=\operatorname{tr}(A B)$.

\subsection{Some tangential operators and spaces}

Let $\mathbf{e}$ be a vector function; we denote by $e_{\Gamma}$ and $w$ the tangential component of $\mathbf{e}$ and its algebraic magnitude on the normal part respectively, i.e.

$$
\begin{aligned}
& w=\langle\mathbf{e}, \nabla b\rangle \\
& e_{\Gamma}=\mathbf{e}-w \nabla b .
\end{aligned}
$$

Vector function e will physically represent the displacement of the mid-surface; its components $e_{\Gamma}$ and $w$ will represent the in-place displacement and the normal deflection respectively. Following the definitions introduced in [5], we will use the subsequent notation. 
Definition 1. We define

$$
\begin{aligned}
& G_{\Gamma} w=\frac{1}{2}\left(\left(\nabla b \otimes \nabla_{\Gamma} w\right) D^{2} b+D^{2} b\left(\nabla_{\Gamma} w \otimes \nabla b\right)\right) \\
& V_{\Gamma} e_{\Gamma}=\frac{1}{2}\left(\left(D^{2} b e_{\Gamma}\right) \otimes \nabla b+\nabla b \otimes\left(D^{2} b e_{\Gamma}\right)\right) \\
& S_{\Gamma} w=\frac{1}{2}\left(D_{\Gamma}^{2} w+{ }^{*} D_{\Gamma}^{2} w\right) .
\end{aligned}
$$

Operator $G_{\Gamma}$ is a first-order tangential operator, $V_{\Gamma}$ is a zero-order tangential operator, and $S_{\Gamma}$ is the symmetrization of the Hessian (which is not we note symmetric in the tangential calculus [11]).

Definition 2. Let us define

$$
\begin{aligned}
& B_{M}(\mathbf{e})=\varepsilon_{\Gamma}\left(e_{\Gamma}\right)+V_{\Gamma} e_{\Gamma}+w D^{2} b+\frac{1}{2} \nabla_{\Gamma} w \otimes \nabla_{\Gamma} w \\
& B_{F}(\mathbf{e})=S_{\Gamma} w+G_{\Gamma} w .
\end{aligned}
$$

Operators $B_{M}$ and $B_{F}$ will correspond to the membrane and bending (flexural) strains respectively. In the rest of this paper the following functional space will be useful.

Definition 3. For $m \in \mathbb{N}$, define the spaces $V^{m}$ as

$$
V^{m}(\Gamma)=\left\{\mathbf{e} \in\left[H^{m}(\Gamma)\right]^{2} \times H^{m+1}(\Gamma) \mid e_{\Gamma}=0, w=0, \frac{\partial}{\partial \nu} w=0 \text { on } \partial \Gamma\right\} .
$$

\section{Statement of the main results}

\subsection{Equation to be studied}

Let $\gamma=l^{2} / 12$, and for a matrix $A$ define operator $\mathcal{C}$ by

$$
\mathcal{C}(A)=\lambda(\operatorname{tr} A) I+2 \mu A .
$$

We consider the following equation in the variable $\mathbf{e}=\left[e_{\Gamma}, w\right]$, where $e_{\Gamma}$ and $w$ are respectively the tangential and vertical displacements and are defined above in Eqs. (10):

$$
\left\{\begin{array}{l}
\rho \partial_{t t} w-\rho \gamma \Delta_{\Gamma} \partial_{t t} w+(\lambda+2 \mu) \gamma \Delta_{\Gamma}^{2} w+\rho \frac{\gamma}{2} \operatorname{div}_{\Gamma}\left(D^{2} b \partial_{t t} e_{\Gamma}\right)+\operatorname{tr}\left(\mathcal{C}\left(\varepsilon_{\Gamma} e_{\Gamma}+V_{\Gamma} e_{\Gamma}\right) D^{2} b\right) \\
\quad+\operatorname{tr}\left(\mathcal{C}\left(w D^{2} b+\frac{1}{2} \nabla_{\Gamma} w \otimes \nabla_{\Gamma} w\right) D^{2} b\right)-\operatorname{div}_{\Gamma}\left(\mathcal{C}\left(\varepsilon_{\Gamma} e_{\Gamma}+V_{\Gamma} e_{\Gamma}\right) \nabla_{\Gamma} w\right) \\
\quad-\operatorname{div}_{\Gamma}\left(\mathcal{C}\left(w D^{2} b+\frac{1}{2} \nabla_{\Gamma} w \otimes \nabla_{\Gamma} w\right) \nabla_{\Gamma} w\right)+2 \mu \gamma \operatorname{div}_{\Gamma}\left(K \nabla_{\Gamma} w\right)+2 \mu \gamma \operatorname{div}_{\Gamma}\left(\left(D^{2} b\right)^{2} \nabla w\right)=0 \\
\rho\left(I+\gamma\left(D^{2} b\right)^{2}\right) \partial_{t t} e_{\Gamma}-\rho \frac{\gamma}{2} D^{2} b \partial_{t t} w-\operatorname{div}_{\Gamma}\left(\mathcal{C}\left(\varepsilon_{\Gamma} e_{\Gamma}+V_{\Gamma} e_{\Gamma}\right)\right) \\
\quad-\operatorname{div}_{\Gamma}\left(\mathcal{C}\left(w D^{2} b+\frac{1}{2} \nabla_{\Gamma} w \otimes \nabla_{\Gamma} w\right)\right)=0
\end{array}\right.
$$

where the derivatives are to be understood in the distributional sense.

Let $k=\lambda H^{2}+\mu\left(H^{2}-2 K\right)$ be a positive real number and $\mathfrak{m}(\mathbf{e}, \hat{\mathbf{e}}), \mathfrak{a}(\mathbf{e}, \hat{\mathbf{e}}), \mathfrak{n}(\mathbf{e}, \hat{\mathbf{e}})$ be defined by

$$
\begin{aligned}
\mathfrak{m}(\mathbf{e}, \hat{\mathbf{e}})= & \rho\left[2\left(e_{\Gamma}, \hat{e_{\Gamma}}\right)_{\Gamma}+2 \gamma\left(\left(D^{2} b\right) e_{\Gamma},\left(D^{2} b\right) \hat{e_{\Gamma}}\right)_{\Gamma}-\gamma\left(\nabla_{\Gamma} w,\left(D^{2} b\right) \hat{e_{\Gamma}}\right)_{\Gamma}\right. \\
& \left.-\gamma\left(\left(D^{2} b\right) e_{\Gamma}, \nabla_{\Gamma} \hat{w}\right)_{\Gamma}+2(w, \hat{w})_{\Gamma}+2 \gamma\left(\nabla_{\Gamma} w, \nabla_{\Gamma} \hat{w}\right)_{\Gamma}\right] \\
\mathfrak{a}(\mathbf{e}, \hat{\mathbf{e}})= & 8 \lambda(k w, k \hat{w})_{\Gamma}+4 \lambda\left(H \operatorname{div}_{\Gamma} e_{\Gamma}, \hat{w}\right)_{\Gamma}+4 \lambda\left(H w, \operatorname{div}_{\Gamma} \hat{e}_{\Gamma}\right)_{\Gamma} \\
& +2 \lambda\left(\operatorname{div}_{\Gamma} e_{\Gamma}, \operatorname{div}_{\Gamma} \hat{e}_{\Gamma}\right)_{\Gamma}+4 \mu \int_{\Gamma} \operatorname{tr}\left(\left(\varepsilon_{\Gamma}\left(e_{\Gamma}\right)+V_{\Gamma} e_{\Gamma}\right)\left(\varepsilon_{\Gamma}\left(\hat{e}_{\Gamma}\right)+V_{\Gamma} \hat{e}_{\Gamma}\right)\right)
\end{aligned}
$$




$$
\begin{aligned}
& +4 \mu \int_{\Gamma} w \operatorname{tr}\left(\left(\varepsilon_{\Gamma}\left(\hat{e}_{\Gamma}\right)+V_{\Gamma} \hat{e}_{\Gamma}\right) D^{2} b\right)+4 \mu \int_{\Gamma} \hat{w} \operatorname{tr}\left(\left(\varepsilon_{\Gamma}\left(e_{\Gamma}\right)+V_{\Gamma} e_{\Gamma}\right) D^{2} b\right) \\
& +2 \gamma \lambda\left(\Delta_{\Gamma} w, \Delta_{\Gamma} \hat{w}\right)_{\Gamma}+4 \gamma \mu \int_{\Gamma} \operatorname{tr}\left(\left(S_{\Gamma} w+G_{\Gamma} w\right)\left(S_{\Gamma} \hat{w}+G_{\Gamma} \hat{w}\right)\right) \\
-\mathfrak{n}(\mathbf{e}, \hat{\mathbf{e}})= & 2 \lambda\left(H\left\|\nabla_{\Gamma} w\right\|^{2}, \hat{w}\right)_{\Gamma}+\lambda\left(\left\|\nabla_{\Gamma} w\right\|^{2}, \operatorname{div}_{\Gamma} \hat{e}_{\Gamma}\right)_{\Gamma} \\
& +4 \lambda\left(H w \nabla_{\Gamma} w, \nabla_{\Gamma} \hat{w}\right)_{\Gamma}+2 \lambda\left(\operatorname{div}_{\Gamma} e_{\Gamma} \nabla_{\Gamma} w, \nabla_{\Gamma} \hat{w}\right)_{\Gamma} \\
& +(\lambda+2 \mu)\left(\left\|\nabla_{\Gamma} w\right\|^{2} \nabla_{\Gamma} w, \nabla_{\Gamma} \hat{w}\right)_{\Gamma}+2 \mu \int_{\Gamma} \operatorname{tr}\left(\varepsilon_{\Gamma}\left(\hat{e}_{\Gamma}\right)\left(\nabla_{\Gamma} w \otimes \nabla_{\Gamma} w\right)\right) \\
& +2 \mu \int_{\Gamma} \hat{w} \operatorname{tr}\left(D^{2} b\left(\nabla_{\Gamma} w \otimes \nabla_{\Gamma} w\right)\right)+4 \mu \int_{\Gamma} w\left\langle D^{2} b \nabla_{\Gamma} w, \nabla_{\Gamma} \hat{w}\right\rangle \\
& +4 \mu \int_{\Gamma} \operatorname{tr}\left(\left(\nabla_{\Gamma} w \otimes \nabla_{\Gamma} \hat{w}\right)\left(\varepsilon_{\Gamma}\left(e_{\Gamma}\right)+V_{\Gamma} e_{\Gamma}\right)\right) .
\end{aligned}
$$

The variational problem associated with (12) is, for each $t \in\left[0, \tau\left[\right.\right.$ and all test functions $\hat{\mathbf{e}} \in V^{1}(\Gamma)$,

$$
\mathfrak{m}\left(\partial_{t t} \mathbf{e}, \hat{\mathbf{e}}\right)+\mathfrak{a}(\mathbf{e}, \hat{\mathbf{e}})-\mathfrak{n}(\mathbf{e}, \hat{\mathbf{e}})=0
$$

where $\mathbf{e}(0)=\mathbf{e}^{0}$ and $\partial_{t} \mathbf{e}(0)=\mathbf{e}^{1}$.

Definition 4 (Weak Solutions). We say that e $\in C^{0}\left(\left[0, \tau\left[, V^{1}(\Gamma)\right) \cap C^{1}\left(\left[0, \tau\left[, V^{0}(\Gamma)\right)\right.\right.\right.\right.$ is a weak solution to the small finite deflections shell problem if and only if (16) is satisfied.

Definition 5 (Regular Solutions). We say that $\mathbf{e}$ is a regular solution to the small finite deflections shell problem if and only if it is a weak solution (i.e. satisfies Eq. (16)) and $\mathbf{e} \in C^{0}\left(\left[0, T\left[, V^{2}(\Gamma)\right) \cap C^{1}\left(\left[0, \tau\left[, V^{1}(\Gamma)\right) \cap C^{2}\left(\left[0, \tau\left[, V^{0}(\Gamma)\right)\right.\right.\right.\right.\right.\right.$.

Remark 1. In the special case of a plate, the curvature tensor $D^{2} b$ vanishes so all the terms including it, like $G_{\Gamma}, V_{\Gamma}$, $K$, vanish as well; we obtain:

$$
\left\{\begin{array}{l}
\rho \partial_{t t} w-\rho \gamma \Delta_{\Gamma} \partial_{t t} w+(\lambda+2 \mu) \gamma \Delta_{\Gamma}^{2} w-\operatorname{div}_{\Gamma}\left(\mathcal{C}\left(\varepsilon_{\Gamma} e_{\Gamma}+\frac{1}{2} \nabla_{\Gamma} w \otimes \nabla_{\Gamma} w\right) \nabla_{\Gamma} w\right)=0 \\
\rho \partial_{t t} e_{\Gamma}-\operatorname{div}_{\Gamma}\left(\mathcal{C}\left(\varepsilon_{\Gamma} e_{\Gamma}\right)+\frac{1}{2} \nabla_{\Gamma} w \otimes \nabla_{\Gamma} w\right)=0
\end{array}\right.
$$

which is the full von Kármán system; see [24].

\subsection{Operators}

Definition 6. Let $\mathcal{M}, \mathcal{A}$ be the linear operators defined by

$$
(\mathcal{M} \mathbf{e}, \hat{\mathbf{e}})=\mathfrak{m}(\mathbf{e}, \hat{\mathbf{e}}) ; \quad(\mathcal{A} \mathbf{e}, \hat{\mathbf{e}})=\mathfrak{a}(\mathbf{e}, \hat{\mathbf{e}}),
$$

let

$$
\mathcal{H}=\mathcal{D}\left(\mathcal{A}^{1 / 2}\right) \times \mathcal{D}\left(\mathcal{M}^{1 / 2}\right)=V^{1}(\Gamma) \times V^{0}(\Gamma)=\left[H^{1}(\Gamma)\right]^{2} \times H^{2}(\Gamma) \times\left[\left(L^{2}\right)(\Gamma)\right]^{2} \times H^{1}(\Gamma)
$$

and let $\mathbb{A}: \mathcal{H} \rightarrow \mathcal{H}$ be defined by

$$
\begin{aligned}
& \mathbb{A}=\left(\begin{array}{cc}
0 & -I \\
\mathcal{M}^{-1} \mathcal{A} & 0
\end{array}\right) \\
& D(A)=\left\{\left(e_{1}, e_{2}\right) \in \mathcal{D}\left(\mathcal{A}^{1 / 2}\right) \times \mathcal{D}\left(\mathcal{A}^{1 / 2}\right), \mathcal{A} e_{1} \in\left[\mathcal{D}\left(\mathcal{M}^{1 / 2}\right)\right]^{\prime}\right\} .
\end{aligned}
$$

Remark 2. A more compact but less explicit expression of the weak formulation can be given by

$$
\frac{1}{2}\left(\mathcal{M} \partial_{t t} \mathbf{e}, \hat{\mathbf{e}}\right)_{\Gamma}+\int_{\Gamma}\left(\mathcal{C} B_{M}(\mathbf{e})\right) \cdots B_{M}^{\prime}(\mathbf{e}, \hat{\mathbf{e}})+\gamma \int_{\Gamma}\left(\mathcal{C} B_{F}(\mathbf{e})\right) \cdots B_{F}(\hat{\mathbf{e}})=0, \quad \forall \hat{\mathbf{e}} \in V^{1}(\Gamma),
$$


where

$$
B_{M}^{\prime}(\mathbf{e}, \hat{\mathbf{e}})=\left.\frac{\partial B_{M}(\mathbf{e}+\theta \hat{\mathbf{e}})}{\partial \theta}\right|_{\theta=0}=\varepsilon_{\Gamma} \hat{e_{\Gamma}}+V_{\Gamma} \hat{e_{\Gamma}}+\hat{w} D^{2} b+\frac{1}{2} \nabla_{\Gamma} w \otimes \nabla_{\Gamma} \hat{w}+\frac{1}{2} \nabla_{\Gamma} \hat{w} \otimes \nabla_{\Gamma} w .
$$

This compact expression (19) will be used in the proof of the existence of regular solutions (Section 6), while the more detailed expression will be used in the proof of the uniqueness (Section 7).

Definition 7. Let $N$ be the nonlinear operator defined by $(N(\mathbf{e}), \hat{\mathbf{e}})=\mathfrak{n}(\mathbf{e}, \hat{\mathbf{e}})$ and $N_{\Gamma}, N_{n}$ be respectively the vector function and scalar function defined by

$$
\begin{aligned}
& N_{\Gamma}(w)=-\lambda \nabla_{\Gamma}\left(\left\|\nabla_{\Gamma} w \Gamma\right\|^{2}\right)+2 \mu \operatorname{div}_{\Gamma}\left(\nabla_{\Gamma} w \otimes \nabla_{\Gamma} w\right) \\
& N_{n}\left(e_{\Gamma}, w\right)=-2 \lambda H\left\|\nabla_{\Gamma} w\right\|^{2}+4 \lambda \operatorname{div}_{\Gamma}\left(H w \nabla_{\Gamma} w\right)+4 \mu \operatorname{div}_{\Gamma}\left(w D^{2} b \nabla_{\Gamma} w\right) \\
&+(\lambda+2 \mu) \operatorname{div}_{\Gamma}\left(\left\|\nabla_{\Gamma} w\right\|^{2} \nabla_{\Gamma} w\right)-2 \mu\left\langle D^{2} b \nabla_{\Gamma} w, \nabla_{\Gamma} w\right\rangle \\
&-2 \lambda \operatorname{div}_{\Gamma}\left(\operatorname{div}_{\Gamma} e_{\Gamma} \nabla_{\Gamma} w\right)-4 \mu \operatorname{div}_{\Gamma}\left(\left(\varepsilon_{\Gamma} e_{\Gamma}+V_{\Gamma} e_{\Gamma}\right) \nabla_{\Gamma} w\right)
\end{aligned}
$$

so we have $N(\mathbf{e})=N_{\Gamma}(w)+N_{n}\left(e_{\Gamma}, w\right) \nabla b$.

It follows, from (16), that:

$$
\partial_{t}\left(\begin{array}{c}
\mathbf{e} \\
\partial_{t} \mathbf{e}
\end{array}\right)+\mathbb{A}\left(\begin{array}{c}
\mathbf{e} \\
\partial_{t} \mathbf{e}
\end{array}\right)=\left(\begin{array}{c}
0 \\
\mathcal{M}^{-1} N(\mathbf{e})
\end{array}\right) .
$$

\subsection{Main results}

Our main results are:

Theorem 1 (Regular Solutions). We consider Eq. (16). Assume that $\mathbf{e}(0) \in V^{2}(\Gamma)$ and $\partial_{t} \mathbf{e}(0) \in V^{1}(\Gamma)$. Then, there exists a unique regular global solution

$$
\mathbf{e} \in C\left(\left[0, \tau\left[; V^{2}(\Gamma)\right) \cap C^{1}\left(\left[0, \tau\left[; V^{1}(\Gamma)\right) \cap C^{2}\left(\left[0, \tau\left[; V^{0}(\Gamma)\right)\right.\right.\right.\right.\right.\right.
$$

where $\tau>0$ is arbitrary.

Theorem 2 (Existence and Uniqueness of Weak Solutions). We consider Eq. (16). Assume that $\mathbf{e}(0) \in V^{1}(\Gamma)$ and $\partial_{t} \mathbf{e}(0) \in V^{0}(\Gamma)$. Then there exists a weak solution

$$
\mathbf{e} \in C\left(\left[0, \tau\left[; V^{1}(\Gamma)\right) \cap C^{1}\left(\left[0, \tau\left[; V^{0}(\Gamma)\right)\right.\right.\right.\right.
$$

which is unique, where $\tau>0$ is arbitrary.

Theorem 3 (Continuous Dependence w.r.t. Initial Data). Weak solutions to Eq. (16) depend continuously on the initial data in the finite energy norm. That is to say, for all $\tau>0$ and all sequences of initial data such that

$$
\begin{aligned}
& \mathbf{e}^{n}(t) \rightarrow \mathbf{e}^{0} \quad \text { in } V^{1}(\Gamma) \\
& \partial_{t} \mathbf{e}^{n}(t) \rightarrow \mathbf{e}^{1} \quad \text { in } V^{0}(\Gamma)
\end{aligned}
$$

the corresponding solutions $\mathbf{e}^{n}(t) \in C\left(\left[0, \tau\left[; V^{1}(\Gamma)\right)\right.\right.$ and $\mathbf{e}(t) \in C\left(\left[0, \tau\left[; V^{1}(\Gamma)\right)\right.\right.$ satisfy:

$$
\begin{aligned}
& \mathbf{e}^{n} \rightarrow \mathbf{e} \quad \text { in } C\left(\left[0, \tau\left[; V^{1}(\Gamma)\right)\right.\right. \\
& \partial_{t} \mathbf{e}^{n} \rightarrow \partial_{t} \mathbf{e} \quad \text { in } C\left(\left[0, \tau\left[; V^{0}(\Gamma)\right) .\right.\right.
\end{aligned}
$$

As a consequence we obtain an energy identity for weak solutions.

The result presented in Theorem 1 is standard for an equation of the type of (16), though no references seem to be available for the proof this particular result. Theorems 2 and 3 are new and solve an old problem in the field.

The reminder of the paper is devoted to the proofs of the three results stated above. Theorem 1 is proved in Section 6. Theorem 2 is proved in Section 7. Theorem 3 is proved in Section 8. 


\section{Derivation of the model}

This section is devoted to deriving the model that leads to Eq. (12), which justifies the interest raised by this equation in the first place. The model will be obtained using Hamilton's principle. The next two subsections are devoted to computing the energies. In Section 4.3 Hamilton's principle is used to get the weak formulation, and in Section 4.4 the PDE formulation is derived. Finally in Section 4.5, the total energy of the system is computed and the system is proved to be conservative (Proposition 1).

The reader solely interested in the Hadamard well-posedness of (12) may skip this section as notation introduced herein is explicitly referenced in the next sections and Proposition 1 is fairly standard.

Since the deflections are not infinitesimal, the strain-displacement relationship is not assumed to be purely linear, and instead is of the form given in Eq. (27) (see [9,22]). The displacement of the shell $T$ and the displacement of the mid-surface $e$ are related by the Kirchhoff hypothesis, that is that the filaments of the plate initially perpendicular to the middle surface remain straight and perpendicular to the deformed surface, and undergo neither contraction nor extension. Following [5], we have

$$
T=\mathbf{e} \circ p-b\left({ }^{*} D_{\Gamma} \mathbf{e} \nabla b\right) \circ p
$$

which leads to

$$
T=e_{\Gamma} \circ p+(w \nabla b) \circ p+b\left(D^{2} b e_{\Gamma}-\nabla_{\Gamma} w\right) \circ p .
$$

\subsection{The kinetic energy}

The kinetic energy is given by

$$
\mathcal{E}_{k}(\mathbf{e})=\frac{\rho}{2} \int_{\mathcal{S}_{l}}\left|\partial_{t} T\right|^{2} .
$$

Following [5], the kinetic energy of the system at any time $t$ is given by

$$
\mathcal{E}_{k}(\mathbf{e})=\frac{\rho l}{2} \int_{\Gamma}\left|\partial_{t} e_{\Gamma}\right|^{2}+\left|\partial_{t} w\right|^{2}+\frac{\rho l \gamma}{2} \int_{\Gamma}\left|D^{2} b \partial_{t} e_{\Gamma}\right|^{2}+\left|\nabla_{\Gamma} \partial_{t} w\right|^{2}-\left\langle D^{2} b \partial_{t} e_{\Gamma}, \nabla_{\Gamma} \partial_{t} w\right\rangle .
$$

\subsection{The elastic energy}

The key hypothesis, and main difference from the model studied in [5], is the strain-displacement relations

$$
\varepsilon(T)=\frac{1}{2}\left({ }^{*} D T+D T\right)+\frac{1}{2} * D T D T .
$$

Following [21, Section 11], several parts of the nonlinear term ${ }^{*} D T D T$ will be neglected; this will in particular be the case for all the terms involving ${ }^{*} D_{\Gamma} e_{\Gamma}$ that appear in ${ }^{*} D T D T$. This is consistent with the shallowness assumption (Hypothesis (ii), Section 1), as these nonlinear terms appear in combination with higher order derivatives of the oriented distance function $b$. Eqs. (23) and (27) yield

$$
\varepsilon(T)=\left(\varepsilon_{\Gamma}\left(e_{\Gamma}\right)+w D^{2} b+V_{\Gamma} e_{\Gamma}+\frac{1}{2} \nabla_{\Gamma} w \otimes \nabla_{\Gamma} w+\frac{1}{2}\left\|\nabla_{\Gamma} w\right\|^{2} \nabla b \otimes \nabla b\right) \circ p-b\left(S_{\Gamma} w+G_{\Gamma} w\right) \circ p .
$$

From now on, and when no confusion is possible, we will write $\varepsilon$ instead of $\varepsilon(T)$.

Assuming isotropy and homogeneity of the material, one can apply Hooke's law to give the elastic energy:

$$
\mathcal{E}_{p}(\mathbf{e})=\frac{1}{2} \int_{\mathcal{S}_{l}} \mathcal{C}(\varepsilon) \cdots \varepsilon
$$


At this point in the computation of the elastic energy, it is customary to impose the hypothesis of plane stresses: $\sigma \cdots(\nabla b \otimes \nabla b)=0$ (which in local coordinates is denoted as $\sigma_{33}=0$ ). In order to satisfy this requirement, we change $\varepsilon(T)$ to

$$
\varepsilon(T)=\left(\varepsilon_{\Gamma}\left(e_{\Gamma}\right)+w D^{2} b+V_{\Gamma} e_{\Gamma}+\frac{1}{2} \nabla_{\Gamma} w \otimes \nabla_{\Gamma} w\right) \circ p-b\left(S_{\Gamma} w+G_{\Gamma} w\right) \circ p .
$$

With the notation of Definition 2 we have

$$
\varepsilon(T)=\left(B_{M}(\mathbf{e})\right) \circ p+b\left(B_{F}(\mathbf{e})\right) \circ p .
$$

This new form of $\varepsilon(T)$ is in line with [2, page 38, Section 7.3]. Eq. (29) yields

$$
\begin{aligned}
\mathcal{E}_{p}(\mathbf{e})= & \frac{1}{2} \int_{\mathcal{S}_{l}}\left[\mathcal{C}\left(B_{M}(\mathbf{e})\right) \cdots B_{M}(\mathbf{e})\right] \circ p+\frac{1}{2} \int_{\mathcal{S}_{l}} b\left[\mathcal{C}\left(B_{M}(\mathbf{e})\right) \cdots B_{F}(\mathbf{e})+\mathcal{C}\left(B_{F}(\mathbf{e})\right) \cdots B_{M}(\mathbf{e})\right] \circ p \\
& +\frac{1}{2} \int_{\mathcal{S}_{l}} b^{2}\left[\mathcal{C}\left(B_{F}(\mathbf{e})\right) \cdots B_{F}(\mathbf{e})\right] \circ p .
\end{aligned}
$$

We define

$$
\begin{aligned}
& \mathcal{E}_{p}^{M}(\mathbf{e})=\frac{1}{2} \int_{\mathcal{S}_{l}}\left[\mathcal{C}\left(B_{M}(\mathbf{e})\right) \cdots B_{M}(\mathbf{e})\right] \circ p \\
& \mathcal{E}_{p}^{F}(\mathbf{e})=\frac{1}{2} \int_{\mathcal{S}_{l}} b^{2}\left[\mathcal{C}\left(B_{F}(\mathbf{e})\right) \cdots B_{F}(\mathbf{e})\right] \circ p
\end{aligned}
$$

as the membrane and bending (flexural) energy, respectively. Since the second integral of the right hand side of (31) vanishes, we have

$$
\mathcal{E}_{p}(\mathbf{e})=\mathcal{E}_{p}^{M}(\mathbf{e})+\mathcal{E}_{p}^{F}(\mathbf{e}) .
$$

We compute the explicit form of the bending energy:

$$
\begin{aligned}
\mathcal{E}_{p}^{F}(\mathbf{e}) & =\frac{1}{2} \int_{\mathcal{S}_{l}} b^{2}\left[\mathcal{C}\left(B_{F}(\mathbf{e})\right) \cdots B_{F}(\mathbf{e})\right] \circ p \\
& =\frac{\lambda}{2} \int_{\mathcal{S}_{l}} b^{2}\left(\operatorname{tr}\left(B_{F}(\varepsilon)\right)\right)^{2} \circ p+\mu \int_{\mathcal{S}_{l}} b^{2} \operatorname{tr}\left(\left(B_{F} \varepsilon\right)^{2}\right) \circ p \\
& =\frac{\lambda}{2} \int_{-l / 2}^{l / 2} z^{2} \int_{\Gamma^{z}}\left(\operatorname{tr}\left(B_{F}(\varepsilon)\right)\right)^{2}+\mu \int_{-l / 2}^{l / 2} z^{2} \int_{\Gamma^{z}} \operatorname{tr}\left(\left(B_{F} \varepsilon\right)^{2}\right) \\
& =\frac{\lambda l^{3}}{24} \int_{\Gamma}\left(\operatorname{tr}\left(B_{F}(\varepsilon)\right)\right)^{2}+\frac{\mu l^{3}}{12} \int_{\Gamma} \operatorname{tr}\left(\left(B_{F} \varepsilon\right)^{2}\right) .
\end{aligned}
$$

Using $\operatorname{tr} G_{\Gamma} w=0$ and $\operatorname{tr} S_{\Gamma} w=\Delta_{\Gamma} w$ we get

$$
\mathcal{E}_{p}^{F}(\mathbf{e})=\frac{\lambda l^{3}}{24}\left\|\Delta_{\Gamma} w\right\|_{L_{2}(\Gamma)}^{2}+\frac{\mu l^{3}}{12} \int_{\Gamma} \operatorname{tr}\left(\left(S_{\Gamma} w+G_{\Gamma} w\right)^{2}\right) .
$$

Analogous computations can be carried out with $\mathcal{E}_{p}^{M}(\mathbf{e})$. We use that $D^{2} b \nabla b=0$ and that for any vector $u$ we have $\operatorname{tr} V_{\Gamma} u=0$ and $\operatorname{tr}\left(V_{\Gamma} u D^{2} b\right)=0$ for any vector $u$. We also use that $\operatorname{tr}\left(\nabla_{\Gamma} w \otimes \nabla_{\Gamma} w\right)=\left\|\nabla_{\Gamma} w\right\|^{2}$ and that $\Delta b=\operatorname{tr} D^{2} b=2 H$. We obtain that the elastic energy of the system is given by

$$
\begin{aligned}
\mathcal{E}_{p}(\mathbf{e})= & \frac{\lambda l}{2}\left|2 H w+\operatorname{div}_{\Gamma} e_{\Gamma}+\frac{1}{2}\left\|\nabla_{\Gamma} w\right\|^{2}\right|_{0, \Gamma}^{2} \\
& +\mu l \int_{\Gamma} \operatorname{tr}\left[\left(\varepsilon_{\Gamma}\left(e_{\Gamma}\right)+D^{2} b w+V_{\Gamma} e_{\Gamma}+\frac{1}{2} \nabla_{\Gamma} w \otimes \nabla_{\Gamma} w\right)^{2}\right]
\end{aligned}
$$




$$
+\frac{\lambda l^{3}}{24}\left|\Delta_{\Gamma} w\right|_{0, \Gamma}^{2}+\frac{\mu l^{3}}{12} \int_{\Gamma} \operatorname{tr}\left[\left(S_{\Gamma} w+G_{\Gamma} w\right)^{2}\right] .
$$

\subsection{Weak formulation of the model}

Let us consider a final time $\tau>0$. Among all kinematically admissible displacements, the actual motion of the shell will make stationary the Lagrangian

$$
\mathcal{L}(\mathbf{e})=\int_{0}^{\tau} \mathcal{E}_{k}(\mathbf{e})-\mathcal{E}_{p}(\mathbf{e})=\int_{0}^{\tau} \mathcal{E}_{k}(\mathbf{e})-\mathcal{E}_{p}^{M}(\mathbf{e})-\mathcal{E}_{p}^{F}(\mathbf{e}) .
$$

We consider the Gâteaux derivative in a direction $\hat{\mathbf{e}}=\hat{e}_{\Gamma}+\hat{w} \nabla b$; we have

$$
\forall \hat{\mathbf{e}} \quad,\left.\frac{\partial \mathcal{L}(\mathbf{e}+\theta \hat{\mathbf{e}})}{\partial \theta}\right|_{\theta=0}=0 .
$$

Note that $S$ symmetric implies $S \cdots M=S \cdots\left({ }^{*} M+M\right)$ so the two last terms of $B_{M}^{\prime}(\mathbf{e}, \hat{\mathbf{e}})$ will often be replaced by twice one of them.

$$
\begin{aligned}
& \left.\frac{\partial \mathcal{E}_{p}^{M}(\mathbf{e}+\theta \hat{\mathbf{e}})}{\partial \theta}\right|_{\theta=0}=\int_{\Gamma}\left(\mathcal{C} B_{M}(\mathbf{e})\right) \cdots B_{M}^{\prime}(\mathbf{e}, \hat{\mathbf{e}}) \\
& \left.\frac{\partial \mathcal{E}_{p}^{F}(\mathbf{e}+\theta \hat{\mathbf{e}})}{\partial \theta}\right|_{\theta=0}=\gamma \int_{\Gamma}\left(\mathcal{C} B_{F}(\mathbf{e})\right) \cdots B_{F}(\hat{\mathbf{e}})
\end{aligned}
$$

and it follows that

$$
\begin{aligned}
\left.\frac{\partial \mathcal{E}_{p}(\mathbf{e}+\theta \hat{\mathbf{e}})}{\partial \theta}\right|_{\theta=0}= & \int_{\Gamma}\left(\mathcal{C} B_{M}(\mathbf{e})\right) \cdots B_{M}^{\prime}(\mathbf{e}, \hat{\mathbf{e}})+\gamma \int_{\Gamma}\left(\mathcal{C} B_{F}(\mathbf{e})\right) \cdots B_{F}(\hat{\mathbf{e}}) \\
= & \lambda l \int_{\Gamma}\left(2 H w+\operatorname{div}_{\Gamma} e_{\Gamma}+\frac{1}{2}\left\|\nabla_{\Gamma} w\right\|^{2}\right)\left(2 H \hat{w}+\operatorname{div}_{\Gamma} \hat{e}_{\Gamma}+\left\langle\nabla_{\Gamma} w, \nabla_{\Gamma} \hat{w}\right\rangle\right) \\
& +2 \mu l \int_{\Gamma} \operatorname{tr}\left(\left(\varepsilon_{\Gamma} e_{\Gamma}+D^{2} b w+V_{\Gamma} e_{\Gamma}+\frac{1}{2} \nabla_{\Gamma} w \otimes \nabla_{\Gamma} w\right)\right. \\
& \left.\times\left(\varepsilon_{\Gamma} \hat{e}_{\Gamma}+D^{2} b \hat{w}+V_{\Gamma} \hat{e}_{\Gamma}+\frac{1}{2} \nabla_{\Gamma} \hat{w} \otimes \nabla_{\Gamma} w+\frac{1}{2} \nabla_{\Gamma} w \otimes \nabla_{\Gamma} \hat{w}\right)\right) \\
& +\frac{\lambda l^{3}}{12} \int_{\Gamma} \Delta_{\Gamma} w \Delta_{\Gamma} \hat{w}+\frac{\mu l^{3}}{6} \int_{\Gamma} \operatorname{tr}\left(\left(S_{\Gamma} w+G_{\Gamma} w\right)\left(S_{\Gamma} \hat{w}+G \hat{w}\right)\right) .
\end{aligned}
$$

Consequently, if $\mathbf{e}$ is an admissible displacement of the mid-surface of the shell which is sufficiently regular then the following expression vanishes for any $\hat{\mathbf{e}}=\hat{e}_{\Gamma}+\hat{w} \nabla b \in V^{1}(\Gamma)$

$$
\begin{aligned}
\int_{0}^{\tau} & \int_{\Gamma}-2 \rho\left\langle\partial_{t} e_{\Gamma}, \partial_{t} \hat{e}_{\Gamma}\right\rangle-2 \rho \partial_{t} w \partial_{t} \hat{w}+\gamma \int_{0}^{\tau} \int_{\Gamma}-2 \rho\left\langle D^{2} b \partial_{t} e_{\Gamma}, D^{2} b \partial_{t} \hat{e}_{\Gamma}\right\rangle-2 \rho\left\langle\nabla_{\Gamma} \partial_{t} w, \nabla_{\Gamma} \partial_{t} \hat{w}\right\rangle \\
& +\rho\left\langle D^{2} b \partial_{t} e_{\Gamma}, \nabla_{\Gamma} \partial_{t} \hat{w}\right\rangle+\rho\left\langle D^{2} b \partial_{t} \hat{e}_{\Gamma}, \nabla_{\Gamma} \partial_{t} w\right\rangle \\
& +\int_{0}^{\tau} \int_{\Gamma} 8 \lambda H^{2} w \hat{w}+4 \lambda H \operatorname{div}_{\Gamma} e_{\Gamma} \hat{w}+\lambda H\left\|\nabla_{\Gamma} w\right\|^{2} \hat{w} \\
& +4 \lambda H w \operatorname{div}_{\Gamma} \hat{e}_{\Gamma}+2 \lambda \operatorname{div}_{\Gamma} e_{\Gamma} \operatorname{div}_{\Gamma} \hat{e}_{\Gamma}+2 \lambda\left\|\nabla_{\Gamma} w\right\|^{2} \operatorname{div}_{\Gamma} \hat{e}_{\Gamma} \\
& +4 \lambda H w\left\langle\nabla_{\Gamma} w, \nabla_{\Gamma} \hat{w}\right\rangle+2 \lambda \operatorname{div}_{\Gamma} e_{\Gamma}\left\langle\nabla_{\Gamma} w, \nabla_{\Gamma} \hat{w}\right\rangle \\
& +\lambda\left\langle\nabla_{\Gamma} w, \nabla_{\Gamma} \hat{w}\right\rangle\left\|\nabla_{\Gamma} w\right\|^{2}+4 \mu \operatorname{tr}\left(\left(\varepsilon_{\Gamma}\left(e_{\Gamma}\right)+V_{\Gamma} e_{\Gamma}\right)\left(\varepsilon_{\Gamma}\left(\hat{e}_{\Gamma}\right)+V_{\Gamma} \hat{e}_{\Gamma}\right)\right) \\
& +4 \mu w \operatorname{tr}\left(\left(\varepsilon_{\Gamma}\left(\hat{e}_{\Gamma}\right)+V_{\Gamma} \hat{e}_{\Gamma}\right) D^{2} b\right)+4 \mu \hat{w} \operatorname{tr}\left(\left(\varepsilon_{\Gamma}\left(e_{\Gamma}\right)+V_{\Gamma} e_{\Gamma}\right) D^{2} b\right)+8 \mu\left(H^{2}-2 K\right) w \hat{w} \\
& +2 \mu\left\|\nabla_{\Gamma} w\right\|^{2}\left\langle\nabla_{\Gamma} w, \nabla_{\Gamma} \hat{w}\right\rangle+2 \mu \operatorname{tr}\left(\left(\varepsilon_{\Gamma}\left(\hat{e}_{\Gamma}\right)+V_{\Gamma} \hat{e}_{\Gamma}\right)\left(\nabla_{\Gamma} w \otimes \nabla_{\Gamma} w\right)\right)+2 \mu \hat{w} \operatorname{tr}\left(D^{2} b\left(\nabla_{\Gamma} w \otimes \nabla_{\Gamma} w\right)\right) \\
& +2 \mu \operatorname{tr}\left(\left(\nabla_{\Gamma} \hat{w} \otimes \nabla_{\Gamma} w+\nabla_{\Gamma} w \otimes \nabla_{\Gamma} \hat{w}\right)\left(\varepsilon_{\Gamma}\left(e_{\Gamma}\right)+V_{\Gamma} e_{\Gamma}\right)\right)
\end{aligned}
$$


$+2 \mu w \operatorname{tr}\left(\left(\nabla_{\Gamma} \hat{w} \otimes \nabla_{\Gamma} w+\nabla_{\Gamma} w \otimes \nabla_{\Gamma} \hat{w}\right) D^{2} b\right)$

$+\gamma \int_{0}^{\tau} \int_{\Gamma} 2 \lambda\left(\Delta_{\Gamma} w\right)\left(\Delta_{\Gamma} \hat{w}\right)+4 \mu \operatorname{tr}\left(\left(S_{\Gamma} w+G_{\Gamma} w\right)\left(S_{\Gamma} \hat{w}+G_{\Gamma} \hat{w}\right)\right)$.

Therefore, for all $\hat{e}_{\Gamma} \in H_{0}^{1}(\Gamma)$, we have

$$
\begin{aligned}
& \int_{0}^{\tau} \int_{\Gamma}-2 \rho\left\langle\partial_{t} e_{\Gamma}, \partial_{t} \hat{e}_{\Gamma}\right\rangle+\gamma \int_{0}^{\tau} \int_{\Gamma}-2 \rho\left\langle D^{2} b \partial_{t} e_{\Gamma}, D^{2} b \partial_{t} \hat{e}_{\Gamma}\right\rangle+\rho\left\langle D^{2} b \partial_{t} \hat{e}_{\Gamma}, \nabla_{\Gamma} \partial_{t} w\right\rangle \\
& \quad+4 \lambda H w \operatorname{div}_{\Gamma} \hat{e}_{\Gamma}+2 \lambda \operatorname{div}_{\Gamma} e_{\Gamma} \operatorname{div}_{\Gamma} \hat{e}_{\Gamma}+\lambda\left\|\nabla_{\Gamma} w\right\|^{2} \operatorname{div}_{\Gamma} \hat{e}_{\Gamma} \\
& \quad+4 \mu \operatorname{tr}\left(\left(\varepsilon_{\Gamma}\left(e_{\Gamma}\right)+V_{\Gamma} e_{\Gamma}\right)\left(\varepsilon_{\Gamma}\left(\hat{e}_{\Gamma}\right)+V_{\Gamma} \hat{e}_{\Gamma}\right)\right)+4 \mu w \operatorname{tr}\left(\left(\varepsilon_{\Gamma}\left(\hat{e}_{\Gamma}\right)+V_{\Gamma} \hat{e}_{\Gamma}\right) D^{2} b\right) \\
& \quad+2 \mu \operatorname{tr}\left(\left(\varepsilon_{\Gamma}\left(\hat{e}_{\Gamma}\right)+V_{\Gamma} \hat{e}_{\Gamma}\right)\left(\nabla_{\Gamma} w \otimes \nabla_{\Gamma} w\right)\right)=0
\end{aligned}
$$

and for all $\hat{w} \in H_{0}^{2}(\Gamma) \cap H_{0}^{1}(\Gamma)$ we have

$$
\begin{aligned}
& \int_{0}^{\tau} \int_{\Gamma}-2 \rho \partial_{t} w \partial_{t} \hat{w}+\gamma \int_{0}^{\tau} \int_{\Gamma}-2 \rho\left\langle\nabla_{\Gamma} \partial_{t} w, \nabla_{\Gamma} \partial_{t} \hat{w}\right\rangle+\rho\left\langle D^{2} b \partial_{t} e_{\Gamma}, \nabla_{\Gamma} \partial_{t} \hat{w}\right\rangle \\
& \quad+\int_{0}^{\tau} \int_{\Gamma} 8 \lambda H^{2} w \hat{w}+4 \lambda H \operatorname{div}_{\Gamma} e_{\Gamma} \hat{w}+2 \lambda H\left\|\nabla_{\Gamma} w\right\|^{2} \hat{w} \\
& \quad+4 \lambda H w\left\langle\nabla_{\Gamma} w, \nabla_{\Gamma} \hat{w}\right\rangle+2 \lambda \operatorname{div}_{\Gamma} e_{\Gamma}\left\langle\nabla_{\Gamma} w, \nabla_{\Gamma} \hat{w}\right\rangle \\
& \quad+\lambda\left\langle\nabla_{\Gamma} w, \nabla_{\Gamma} \hat{w}\right\rangle\left\|\nabla_{\Gamma} w\right\|^{2}+4 \mu \hat{w} \operatorname{tr}\left(\left(\varepsilon_{\Gamma}\left(e_{\Gamma}\right)+V_{\Gamma} e_{\Gamma}\right) D^{2} b\right) \\
& \quad+8 \mu\left(H^{2}-2 K\right) w \hat{w}+2 \mu\left\|\nabla_{\Gamma} w\right\|^{2}\left\langle\nabla_{\Gamma} w, \nabla_{\Gamma} \hat{w}\right\rangle+2 \mu \hat{w} \operatorname{tr}\left(D^{2} b\left(\nabla_{\Gamma} w \otimes \nabla_{\Gamma} w\right)\right) \\
& \quad+2 \mu \operatorname{tr}\left(\left(\nabla_{\Gamma} \hat{w} \otimes \nabla_{\Gamma} w+\nabla_{\Gamma} w \otimes \nabla_{\Gamma} \hat{w}\right)\left(\varepsilon_{\Gamma}\left(e_{\Gamma}\right)+V_{\Gamma} e_{\Gamma}\right)\right) \\
& \quad+2 \mu w \operatorname{tr}\left(\left(\nabla_{\Gamma} \hat{w} \otimes \nabla_{\Gamma} w+\nabla_{\Gamma} w \otimes \nabla_{\Gamma} \hat{w}\right) D^{2} b\right) \\
& \quad+\gamma \int_{0}^{\tau} \int_{\Gamma} 2 \lambda\left(\Delta_{\Gamma} w\right)\left(\Delta_{\Gamma} \hat{w}\right)+4 \mu \operatorname{tr}\left(\left(S_{\Gamma} w+G_{\Gamma} w\right)\left(S_{\Gamma} \hat{w}+G_{\Gamma} \hat{w}\right)\right)=0 .
\end{aligned}
$$

These equations are in line with [2, Section 7.3].

\section{Lemma 1. The following identities hold true}

$$
\begin{aligned}
& \operatorname{tr}\left(V_{\Gamma} u \nabla_{\Gamma} v \otimes \nabla_{\Gamma} v\right)=0 \\
& \int_{\Gamma} \operatorname{tr}\left(\varepsilon_{\Gamma} u \nabla_{\Gamma} v \otimes \nabla_{\Gamma} v\right)=-\int_{\Gamma}\left\langle\operatorname{div}_{\Gamma}(\nabla v \otimes \nabla v), u\right\rangle .
\end{aligned}
$$

Proof. The term $\operatorname{tr}\left(V_{\Gamma} u \nabla_{\Gamma} v \otimes \nabla_{\Gamma} v\right)$ can be written as

$$
\begin{aligned}
& \frac{1}{2} \operatorname{tr}\left(\left(\left(D^{2} b u\right) \otimes \nabla b\right)\left(\nabla_{\Gamma} v \otimes \nabla_{\Gamma} v\right)+\left(\nabla b \otimes\left(D^{2} b u\right)\right)\left(\nabla_{\Gamma} v \otimes \nabla_{\Gamma} v\right)\right) \\
& \quad=\frac{1}{2} \operatorname{tr}\left(\left\langle\nabla b, \nabla_{\Gamma} v\right\rangle\left(D^{2} b u\right) \otimes \nabla_{\Gamma} v+\left\langle D^{2} b u, \nabla_{\Gamma} v\right\rangle\left(\nabla b \otimes \nabla_{\Gamma} v\right)\right) \\
& \quad=\frac{1}{2}\left\langle\nabla b, \nabla_{\Gamma} v\right\rangle\left\langle D^{2} b u, \nabla_{\Gamma} v\right\rangle+\frac{1}{2}\left\langle D^{2} b u, \nabla_{\Gamma} v\right\rangle\left\langle\nabla b, \nabla_{\Gamma} v\right\rangle
\end{aligned}
$$

and since $\left\langle\nabla b, \nabla_{\Gamma} v\right\rangle=0$ we obtain the first identity. The second one can be obtained by writing

$$
\int_{\Gamma} \operatorname{tr}\left(\varepsilon_{\Gamma} u \nabla_{\Gamma} v \otimes \nabla_{\Gamma} v\right)=\int_{\Gamma} \varepsilon_{\Gamma} u \cdots(\nabla v \otimes \nabla v)=-\int_{\Gamma}\left\langle\operatorname{div}_{\Gamma}(\nabla v \otimes \nabla v), u\right\rangle
$$

If $A$ is a symmetric matrix then $\operatorname{tr}\left(A B^{*}\right)=\operatorname{tr}(A B)$. Using this equality, Lemma 1 and the calculations above gives that if $\mathbf{e}$ is an admissible displacement of the mid-surface of the shell then it satisfies (16). 


\subsection{PDE formulation of the model}

In this subsection we are interested in showing why (12) governs the displacement of the shell. We go back to Eq. (19). We have

$$
\begin{aligned}
&\left(\mathcal{M} \partial_{t t} \mathbf{e}, \hat{\mathbf{e}}\right)_{\Gamma}=\left(2 \rho\left(I+\gamma\left(D^{2} b\right)^{2}\right) \partial_{t t} e_{\Gamma}-\rho \gamma D^{2} b \partial_{t t} w, \hat{e_{\Gamma}}\right)_{\Gamma} \\
&+\left[2 \rho \partial_{t t} w-2 \rho \gamma \Delta_{\Gamma} \partial_{t t} w+\gamma \rho \operatorname{div}_{\Gamma}\left(D^{2} b \partial_{t t} e_{\Gamma}\right)\right] \hat{w} \\
& \int_{\Gamma} \mathcal{C} B_{M}(\mathbf{e}) \cdots B_{M}^{\prime}(\mathbf{e}, \hat{\mathbf{e}})=\left(-\operatorname{div}_{\Gamma}\left(\mathcal{C} B_{M}(\mathbf{e})\right), \hat{e_{\Gamma}}\right)_{\Gamma}+\left[\operatorname{tr}\left(\mathcal{C}\left(B_{M}(\mathbf{e})\right) D^{2} b\right)-\operatorname{div}_{\Gamma}\left(\mathcal{C}\left(B_{M}(\mathbf{e})\right) \nabla_{\Gamma} w\right)\right] \hat{w} .
\end{aligned}
$$

Using [6, Lemma 2.3], we get

$$
\int_{\Gamma} \mathcal{C} B_{F}(\mathbf{e}) \cdots B_{F}(\hat{\mathbf{e}})=\left[(\lambda+2 \mu) \Delta_{\Gamma}^{2} w+2 \mu \operatorname{div}_{\Gamma}\left(K \nabla_{\Gamma} w\right)+2 \mu \operatorname{div}_{\Gamma}\left(\left(D^{2} b\right)^{2} \nabla_{\Gamma} w\right)\right] \hat{w}
$$

Combining (40)-(42), we obtain

$$
\left\{\begin{array}{c}
\rho \partial_{t t} w-\rho \gamma \Delta_{\Gamma} \partial_{t t} w+(\lambda+2 \mu) \gamma \Delta_{\Gamma}^{2} w+\rho \frac{\gamma}{2} \operatorname{div}_{\Gamma}\left(D^{2} b \partial_{t t} e_{\Gamma}\right)+\operatorname{tr}\left(\mathcal{C}\left(B_{M}(\mathbf{e})\right) D^{2} b\right) \\
-\operatorname{div}_{\Gamma}\left(\mathcal{C}\left(B_{M}(\mathbf{e})\right) \nabla_{\Gamma} w\right)+2 \mu \gamma \operatorname{div}_{\Gamma}\left(K \nabla_{\Gamma} w\right)+2 \mu \gamma \operatorname{div}_{\Gamma}\left(\left(D^{2} b\right)^{2} \nabla_{\Gamma} w\right)=0 \\
\rho\left(I+\gamma\left(D^{2} b\right)^{2}\right) \partial_{t t} e_{\Gamma}-\rho \frac{\gamma}{2} D^{2} b \partial_{t t} w-\operatorname{div}_{\Gamma}\left(\mathcal{C} B_{M}(\mathbf{e})\right)=0 .
\end{array}\right.
$$

The definition of $B_{M}$ (Definition 2) gives the desired result (12).

\subsection{Total energy of the system}

The total energy of the system is given by the sum of the kinetic and potential (elastic) energy:

$$
\begin{aligned}
\mathcal{E}(\mathbf{e})= & \mathcal{E}_{k}(\mathbf{e})+\mathcal{E}_{p}(\mathbf{e}) \\
= & \frac{\rho l}{2} \int_{\Gamma}\left|\partial_{t} e_{\Gamma}\right|^{2}+\left|\partial_{t} w\right|^{2}+\frac{\rho l \gamma}{2} \int_{\Gamma}\left|D^{2} b \partial_{t} e_{\Gamma}\right|^{2}+\left|\nabla_{\Gamma} \partial_{t} w\right|^{2}+\left|D^{2} b \partial_{t} e_{\Gamma}-\nabla_{\Gamma} \partial_{t} w\right|^{2} \\
& +\frac{\lambda l}{2}\left|2 H w+\operatorname{div}_{\Gamma} e_{\Gamma}+\frac{1}{2}\left\|\nabla_{\Gamma} w\right\|^{2}\right|_{0, \Gamma}^{2} \\
& +\mu l \int_{\Gamma} \operatorname{tr}\left[\left(\varepsilon_{\Gamma}\left(e_{\Gamma}\right)+D^{2} b w+V_{\Gamma} e_{\Gamma}+\frac{1}{2} \nabla_{\Gamma} w \otimes \nabla_{\Gamma} w\right)^{2}\right] \\
& +\frac{\lambda l^{3}}{24}\left|\Delta_{\Gamma} w\right|_{0, \Gamma}^{2}+\frac{\mu l^{3}}{12} \int_{\Gamma} \operatorname{tr}\left[\left(S_{\Gamma} w+G_{\Gamma} w\right)^{2}\right] .
\end{aligned}
$$

Note that

$$
\mathcal{E}(\mathbf{e}(t)) \geq C\left(\left\|e_{\Gamma}(t)\right\|_{H^{1}(\Gamma)}^{2}+\left\|\partial_{t} e_{\Gamma}(t)\right\|_{L^{2}(\Gamma)}^{2}+\left\|\partial_{t} w(t)\right\|_{H^{1}(\Gamma)}^{2}+\|w(t)\|_{H^{2}(\Gamma)}^{2}\right) .
$$

The sum of the kinetic energy with the bending (flexural) energy will be of some interest in Section 8. It is defined as

$$
\mathcal{E}^{F}(\mathbf{e})=\mathcal{E}_{k}(\mathbf{e})+\mathcal{E}_{p}^{F}(\mathbf{e})
$$

which can also be written as

$$
\mathcal{E}^{F}(\mathbf{e})=\mathcal{E}(\mathbf{e})-\frac{l}{2} \int_{\Gamma} \mathcal{C}\left(B_{M}(\mathbf{e})\right) \cdots B_{M}(\mathbf{e}) .
$$

Note that the nonlinear terms appear only in $B_{M}$ whose energy terms are in $\mathcal{E}_{p}^{M}$. As a consequence the energy terms in $\mathcal{E}^{F}$ all come from linear terms of the equations. 
Proposition 1. Let $\mathbf{e}$ be a regular solution to the small finite deflections shell problem; then

$$
\forall t \in[0, \tau[, \quad \mathcal{E}(\mathbf{e}(t))=\mathcal{E}(\mathbf{e}(0)) .
$$

Proof. Since e $\in C^{0}\left(\left[0, T\left[, V^{2}(\Gamma)\right) \cap C^{1}\left(\left[0, \tau\left[, V^{1}(\Gamma)\right) \cap C^{2}\left(\left[0, \tau\left[, V^{0}(\Gamma)\right)\right.\right.\right.\right.\right.\right.$ one can replace $\hat{\mathbf{e}}$ by $\partial_{t} \mathbf{e}$ in $(16)$; this yields

$$
\mathfrak{m}\left(\partial_{t t} \mathbf{e}, \partial_{t} \mathbf{e}\right)+\mathfrak{a}\left(\mathbf{e}, \partial_{t} \mathbf{e}\right)-\mathfrak{n}\left(\mathbf{e}, \partial_{t} \mathbf{e}\right)=0
$$

and after some computations, it follows that

$$
\partial_{t}\left[\mathfrak{m}\left(\partial_{t} \mathbf{e}, \mathbf{e}\right)\right]+\int_{\Gamma} \mathcal{C}\left(B_{M}(\mathbf{e})\right) \cdots B_{M}^{\prime}\left(\mathbf{e}, \partial_{t} \mathbf{e}\right)+\gamma \int_{\Gamma} \mathcal{C}\left(B_{F}(\mathbf{e})\right) \cdots B_{F}\left(\partial_{t} \mathbf{e}\right)=0 .
$$

Eqs. (37) yield

$$
\partial_{t} \mathcal{E}_{k}(t)+\partial_{t} \mathcal{E}_{p}^{F}(t)+\partial_{t} \mathcal{E}_{p}^{F}(t)=0
$$

and therefore $\partial_{t} \mathcal{E}(t)=0$, which leads to the desired result.

For the sake of simplicity, we shall not introduce new notation for the energy as a function of time and we shall use $\mathcal{E}(t)\left(\operatorname{resp} . \mathcal{E}_{p}(t), \ldots\right)$ to refer to $\mathcal{E}(\mathbf{e}(t))\left(\operatorname{resp} . \mathcal{E}_{p}(\mathbf{e}(t)), \ldots\right)$.

\section{Preliminary properties}

The remainder of this paper is devoted to the proof of Theorems 1-3. The properties established in this section will be used in the proofs of these theorems.

\subsection{Properties of the operators}

Lemma 2. We have $\langle\mathbb{A} X, X\rangle_{\mathcal{H}}=0$ for all $X \in D(A)$.

Proof. We note that the inner product above is on the space $\mathcal{H}$ which is defined in Eq. (17). Let $X=\left[\mathbf{e}^{1} \mathbf{e}^{2}\right]$. Thus we have

$$
\begin{aligned}
\langle\mathbb{A} X, X\rangle_{\mathcal{D}\left(\mathcal{A}^{1 / 2}\right) \times \mathcal{D}\left(\mathcal{M}^{1 / 2}\right)} & =-\left\langle\mathbf{e}^{2}, \mathbf{e}^{1}\right\rangle_{\mathcal{D}\left(\mathcal{A}^{1 / 2}\right)}+\left\langle\mathcal{M}^{-1} \mathcal{A} \mathbf{e}^{1}, \mathbf{e}^{2}\right\rangle_{\mathcal{D}\left(\mathcal{M}^{1 / 2}\right)} \\
& =-\left\langle\mathcal{A}^{1 / 2} \mathbf{e}^{2}, \mathcal{A}^{1 / 2} \mathbf{e}^{1}\right\rangle_{\left[L_{2}(\Gamma)\right]^{3}}+\left\langle\mathcal{M}^{1 / 2} \mathcal{M}^{-1} \mathcal{A} \mathbf{e}^{1}, \mathcal{M}^{1 / 2} \mathbf{e}^{2}\right\rangle_{\left[L_{2}(\Gamma)\right]^{3}} \\
& =-\left\langle\mathbf{e}^{2}, \mathcal{A} \mathbf{e}^{1}\right\rangle_{\left[L_{2}(\Gamma)\right]^{3}}+\left\langle\mathcal{M}^{1 / 2} \mathcal{M}^{1 / 2} \mathcal{M}^{-1} \mathcal{A} \mathbf{e}^{1}, \mathbf{e}^{2}\right\rangle_{\left[L_{2}(\Gamma)\right]^{3}} \\
& =-\left\langle\mathbf{e}^{2}, \mathcal{A} \mathbf{e}^{1}\right\rangle_{\left[L_{2}(\Gamma)\right]^{3}}+\left\langle\mathcal{A} \mathbf{e}^{1}, \mathbf{e}^{2}\right\rangle_{\left[L_{2}(\Gamma)\right]^{3}}=0 .
\end{aligned}
$$

Lemma 3. The bilinear form $\mathfrak{a}(\mathbf{e}, \hat{\mathbf{e}})$ defined in (14) is elliptic on $V^{1}(\Gamma)$, that is, there exists a constant $C>0$ such that

$$
C\|\mathbf{e}\|_{V^{1}(\Gamma)}^{2} \leq \mathfrak{a}(\mathbf{e}, \mathbf{e}) \quad \forall \mathbf{e} \in V^{1}(\Gamma)
$$

In addition the bilinear form $\mathfrak{m}(\mathbf{e}, \hat{\mathbf{e}})$ is elliptic on the space $V^{0}(\Gamma)$. This gives coercivity of the related linear operators $\mathcal{A}, \mathcal{M}$.

Proof. This inequality for $\mathfrak{a}(\mathbf{e}, \hat{\mathbf{e}})$ is established by Bernadou and Oden [3] (see also [2]) provided the shell is shallow enough (as in Hypothesis (ii), Section 1). Though this model does not exactly satisfy the lemma of rigid body motion, the proof relies on the fact that the shallowness assumption allows us to consider the equations above as some perturbation of the special case of the plate. The corresponding proof for $\mathfrak{m}(\mathbf{e}, \hat{\mathbf{e}})$ is given in [5]. 


\subsection{Norm estimates}

Lemma 4. Let $w \in H^{2}(\Gamma)$; then

$$
\left\|\nabla_{\Gamma} w \otimes \nabla_{\Gamma} w\right\|_{L^{2}(\Gamma)} \leq C\|w\|_{H^{2}(\Gamma)}\|w\|_{H^{1}(\Gamma)} .
$$

Proof. We have

$$
\left\|\nabla_{\Gamma} w \otimes \nabla_{\Gamma} w\right\|_{L^{2}(\Gamma)} \leq\left\|\nabla_{\Gamma} w\right\|_{L^{4}(\Gamma)}^{2} .
$$

Using Sobolev's embedding $H^{1 / 2}(\Gamma) \subset L^{4}(\Gamma)$, we obtain

$$
\left\|\nabla_{\Gamma} w \otimes \nabla_{\Gamma} w\right\|_{L^{2}(\Gamma)} \leq C\left\|\nabla_{\Gamma} w\right\|_{H^{1 / 2}(\Gamma)}^{2}
$$

and the classical interpolation inequality $\left\|\nabla_{\Gamma} w\right\|_{H^{1 / 2}(\Gamma)}^{2} \leq\left\|\nabla_{\Gamma} w\right\|_{H^{1}(\Gamma)}\left\|\nabla_{\Gamma} w\right\|_{L^{2}(\Gamma)}$ yields (49).

Let us denote by $\left\{\varphi_{i}\right\}$ the family of the eigenvectors of the Laplace operator and $\lambda_{i}$ the eigenvalues corresponding to $\varphi_{i}$ (the eigenvalues $\lambda_{n}$ should not to be confused with the Lamé coefficient $\lambda$ ). Let $P_{n}$ be the orthogonal projection on the subspace spanned by the $n$ eigenvectors $\varphi_{0}, \ldots, \varphi_{n-1}$. If $f \in L^{2}$ and $g \in H^{1}$, then the estimate resulting from Sobolev's embedding and the Holder inequality gives [30]:

$$
\begin{aligned}
& \left\|\left(P_{n} f\right) g\right\|_{L^{2}} \leq C \sqrt{\ln \left(1+\lambda_{n}\right)}\|f\|_{L^{2}}\|g\|_{H^{1}} \\
& \left\|\left(P_{n} g\right) f\right\|_{L^{2}} \leq C \sqrt{\ln \left(1+\lambda_{n}\right)}\|f\|_{L^{2}}\|g\|_{H^{1}} \\
& \left\|P_{n} g\right\|_{L^{\infty}} \leq C \sqrt{\ln \left(1+\lambda_{n}\right)}\|g\|_{H^{1}} .
\end{aligned}
$$

We define $Q_{n}=I-P_{n}$.

Remark 3. The sequence $\left(\lambda_{n}\right)$ is increasing and $\lim \lambda_{n}=+\infty$.

Lemma 5. Let $\epsilon>0, \alpha>0$ and $f \in H^{\epsilon+\alpha}$; then

$$
\left\|Q_{n} f\right\|_{H^{\epsilon}}^{2} \leq \frac{1}{\lambda^{\alpha}}\left\|Q_{n} f\right\|_{H^{\epsilon+\alpha}}^{2} .
$$

Proof. We have

$$
Q_{n} f=\sum_{i=n}^{+\infty}\left\langle f, \varphi_{i}\right\rangle \varphi_{i}
$$

and, for $\eta>0$, we have

$$
\left\|Q_{n} f\right\|_{H^{\eta}}^{2}=\sum_{i=n}^{+\infty}\left\langle f, \varphi_{i}\right\rangle^{2} \lambda_{i}^{\eta}
$$

Applying (52) with $\eta=\epsilon$ yields

$$
\left\|Q_{n} f\right\|_{H^{\epsilon}}^{2}=\sum_{i=n}^{+\infty}\left\langle f, \varphi_{i}\right\rangle^{2} \lambda_{i}^{\epsilon} \leq\left(\sup _{i \geq n} \frac{1}{\lambda_{i}^{\alpha}}\right) \sum_{i=n}^{+\infty}\left\langle f, \varphi_{i}\right\rangle^{2} \lambda_{i}^{\epsilon+\alpha} .
$$

Using that $\left(\lambda_{i}\right)$ is an increasing sequence, we obtain

$$
\left\|Q_{n} f\right\|_{H^{\epsilon}}^{2} \leq \frac{1}{\lambda_{n}^{\alpha}} \sum_{i=n}^{+\infty}\left\langle f, \varphi_{i}\right\rangle^{2} \lambda_{i}^{\epsilon+\alpha} .
$$

Applying (52) with $\eta=\epsilon+\alpha$ yields

$$
\left\|Q_{n} f\right\|_{H^{\epsilon}}^{2} \leq \frac{1}{\lambda_{n}^{\alpha}}\left\|Q_{n} f\right\|_{H^{\epsilon+\alpha}}^{2} .
$$


Lemma 6. Let $\epsilon>0, \alpha>0, f \in H^{\epsilon+\alpha}$ and $g \in H^{1}$; then

$$
\left\|\left(Q_{n} f\right) g\right\|_{L^{2}}^{2} \leq \frac{1}{\lambda_{n}^{\alpha}}\|f\|_{H^{\epsilon+\alpha}}^{2}\|g\|_{H^{1}}^{2} .
$$

Proof. We have

$$
\left\|\left(Q_{n} f\right) g\right\|_{L^{2}}^{2} \leq\left\|Q_{n} f\right\|_{H^{\epsilon}}^{2}\|g\|_{H^{1}}^{2} .
$$

Lemma 5 gives

$$
\left\|\left(Q_{n} f\right) g\right\|_{L^{2}}^{2} \leq \frac{1}{\lambda_{n}^{\alpha}}\left\|Q_{n} f\right\|_{H^{\epsilon+\alpha}}^{2}\|g\|_{H^{1}}^{2} .
$$

Using $\left\|Q_{n} f\right\|_{H^{\epsilon+\alpha}}^{2} \leq\|f\|_{H^{\epsilon+\alpha}}^{2}$ we obtain (53).

Proposition 2. Let $\epsilon>0, \alpha>0, f \in H^{\epsilon+\alpha}$ and $g \in H^{1}$; then

$$
\|f g\|_{L^{2}}^{2} \leq C\left(\ln \left(1+\lambda_{n}\right)\|f\|_{L^{2}}^{2}+\frac{1}{\lambda_{n}^{\alpha}}\|f\|_{H^{\epsilon+\alpha}}^{2}\right)\|g\|_{H^{1}}^{2} .
$$

Proof. We have

$$
\|f g\|_{L^{2}}^{2}=\left\|\left(P_{n} f\right) g+\left(Q_{n} f\right) g\right\|_{L^{2}}^{2} \leq\left\|\left(P_{n} f\right) g\right\|_{L^{2}}^{2}+\left\|\left(Q_{n} f\right) g\right\|_{L^{2}}^{2}
$$

applying (50a) to $\left\|\left(P_{n} f\right) g\right\|_{L^{2}}$ and (53) to $\left\|\left(Q_{n} f\right) g\right\|_{L^{2}}$ gives (54).

Corollary 1. Let $\epsilon>0, \alpha>0, v_{1} \in\left(H^{\epsilon+\alpha}\right)^{3}$ and $v_{2} \in\left(H^{1}\right)^{3}$; then

$$
\begin{gathered}
\left\|\left\langle v_{1}, v_{2}\right\rangle\right\|_{L^{2}}^{2} \leq C\left(\ln \left(1+\lambda_{n}\right)\left\|v_{1}\right\|_{L^{2}}^{2}+\frac{1}{\lambda_{n}^{\alpha}}\left\|v_{1}\right\|_{H^{\epsilon+\alpha}}^{2}\right)\left\|v_{2}\right\|_{H^{1}}^{2} \\
\left\|v_{1} \otimes v_{2}\right\|_{L^{2}}^{2} \leq C\left(\ln \left(1+\lambda_{n}\right)\left\|v_{1}\right\|_{L^{2}}^{2}+\frac{1}{\lambda_{n}^{\alpha}}\left\|v_{1}\right\|_{H^{\epsilon+\alpha}}^{2}\right)\left\|v_{2}\right\|_{H^{1}}^{2} .
\end{gathered}
$$

Proof. These inequalities follow from Proposition 2 for each term of $\left\langle v_{1}, v_{2}\right\rangle$ or each component of $v_{1} \otimes v_{2}$.

\section{Proof of Theorem 1 (existence of regular solutions)}

In this section we prove Theorem 1, the existence and uniqueness of regular solutions to the problem (16). We shall use the nonlinear Galerkin method, essentially following the proof in [25] for the full von Kármán plate equation.

\subsection{Semidiscrete approximation}

Let $h$ be a non-negative real number and $V_{h}^{2}(\Gamma)$ be a finite dimensional subspace dense in $V^{2}(\Gamma)$ as defined in (11). We consider the following semidiscrete approximation of (19). Given $\mathbf{e}^{0, h}=\left(e_{\Gamma}^{h, 0}, w^{h, 0}\right) \in V_{h}^{2}(\Gamma)$ and $\mathbf{e}^{h, 1}=\left(e_{\Gamma}^{h, 1}, w^{1, h}\right) \in V_{h}^{1}(\Gamma)$, find $\mathbf{e}^{h}=\left(e_{\Gamma}^{h}, w^{h}\right) \in V_{h}^{2}$ such that

$$
\begin{aligned}
& \left(\mathcal{M} \partial_{t t} \mathbf{e}^{h}, \hat{\mathbf{e}}\right)_{\Gamma}+\int_{\Gamma}\left(\mathcal{C} B_{M}\left(\mathbf{e}^{h}\right)\right) \cdots B_{M}^{\prime}\left(\mathbf{e}^{h}, \hat{\mathbf{e}}\right)+\gamma \int_{\Gamma}\left(\mathcal{C} B_{F}\left(\mathbf{e}^{h}\right)\right) \cdots B_{F}(\hat{\mathbf{e}})=0, \quad \forall \hat{\mathbf{e}} \in V_{h}^{2}(\Gamma) \\
& \mathbf{e}^{h}(0)=\mathbf{e}^{h, 0} \\
& \partial_{t} \mathbf{e}^{h}(0)=\mathbf{e}^{h, 1} .
\end{aligned}
$$

Let us define $E_{h}(t)=\mathcal{E}\left(\mathbf{e}^{h}\right)$ where $\mathcal{E}$ is defined in Eq. (44). Let $E_{h}(t)$ denote the energy computed along the semidiscrete solution $\mathbf{e}^{h}$. Since $\mathbf{e}^{h}(t)$ belongs to $V_{h}^{2}(\Gamma)$ it is a regular solution, so Proposition 1 applies.

$$
E_{h}(t) \leq E_{h}(0) .
$$


Global existence and uniqueness of semidiscrete solutions follows from the fact that the nonlinear terms contained in $B_{M}$ are locally Lipschitz on $V_{h}^{2}(\Gamma)$ together with the standard a priori bound (58). It follows that

$$
\begin{aligned}
& \left\|\partial_{t} w_{h}(t)\right\|_{H^{1}}^{2}+\left\|\partial_{t} e_{\Gamma}(t)\right\|_{L^{2}}^{2}+\left\|w_{h}(t)\right\|_{H^{2}}^{2}+\left\|\partial_{t} e_{\Gamma}(t)\right\|_{H^{1}}^{2} \leq E_{h}(t) \leq E_{h}(0) \\
& \left\|\partial_{t} w_{h}\right\|_{L^{2}} \leq \sqrt{E_{h}(0)}
\end{aligned}
$$

for all $\tau>0$ such that the solution $\mathbf{e}^{h}(t) \in C^{\infty}\left(\left[0, \tau\left[, V_{h}^{2}\right)\right.\right.$.

\subsection{Stability in higher norms}

In the following equations, we shall use $\bar{u}=\partial_{t} u^{h}$. Let us differentiate (57a) with respect to $t$; then the following equation holds true for all tests $\hat{\mathbf{e}} \in V_{h}^{2}$

$$
\left(\mathcal{M} \partial_{t t} \overline{\mathbf{e}}, \hat{\mathbf{e}}\right)+\int_{\Gamma}\left(\mathcal{C} B_{M}^{\prime}\left(\mathbf{e}^{h}, \overline{\mathbf{e}}\right)\right) \cdots B_{M}^{\prime}\left(\mathbf{e}^{h}, \hat{\mathbf{e}}\right)+\gamma \int_{\Gamma}\left(\mathcal{C} B_{F}(\overline{\mathbf{e}})\right) \cdots B_{F}(\hat{\mathbf{e}})=0 .
$$

Replacing the test function by $\partial_{t} \hat{\mathbf{e}}$ in (57a) yields a simplification in the equation above; it results in the following equation holding true for all tests $\hat{\mathbf{e}} \in V_{h}^{2}$

$$
\left(\mathcal{M} \partial_{t t} \overline{\mathbf{e}}, \hat{\mathbf{e}}\right)+\int_{\Gamma} \mathcal{C} B_{M}^{\prime}\left(\mathbf{e}^{h}, \overline{\mathbf{e}}\right) \cdots B_{M}^{\prime}\left(\mathbf{e}^{h}, \hat{\mathbf{e}}\right)+\int_{\Gamma} \mathcal{C} B_{M}\left(\mathbf{e}^{h}\right) \cdots\left(\nabla_{\Gamma} \hat{w} \otimes \nabla_{\Gamma} \bar{w}\right)+\gamma \int_{\Gamma}\left(\mathcal{C} B_{F}(\overline{\mathbf{e}})\right) \cdots B_{F}(\hat{\mathbf{e}})=0 .
$$

We note that the differentiation of the nonlinear quantities results in extra terms in the equation. In order to see them we will write explicitly:

$$
\begin{aligned}
\int_{\Gamma} \mathcal{C} & B_{M}^{\prime}\left(\mathbf{e}^{h}, \overline{\mathbf{e}}\right) \cdots B_{M}^{\prime}\left(\mathbf{e}^{h}, \hat{\mathbf{e}}\right)+\int_{\Gamma} \mathcal{C} B_{M}\left(\mathbf{e}^{h}\right) \cdots\left(\nabla_{\Gamma} \hat{w} \otimes \nabla_{\Gamma} \bar{w}\right) \\
= & \int_{\Gamma} \operatorname{tr}\left[\mathcal{C}\left(\varepsilon_{\Gamma} \overline{e_{\Gamma}}+V_{\Gamma} \overline{e_{\Gamma}}+\bar{w} D^{2} b+\frac{1}{2} \nabla_{\Gamma} w^{h} \otimes \nabla_{\Gamma} \bar{w}+\frac{1}{2} \nabla_{\Gamma} \bar{w} \otimes \nabla_{\Gamma} w^{h}\right)\right. \\
& \left.\times\left(\varepsilon_{\Gamma} \hat{e_{\Gamma}}+V_{\Gamma} \hat{e_{\Gamma}}+\hat{w} D^{2} b+\frac{1}{2} \nabla_{\Gamma} w^{h} \otimes \nabla_{\Gamma} \hat{w}+\frac{1}{2} \nabla_{\Gamma} \hat{w} \otimes \nabla_{\Gamma} w^{h}\right)\right] \\
& +\int_{\Gamma} \operatorname{tr}\left[\mathcal{C}\left(\varepsilon_{\Gamma}\left(e_{\Gamma}\right)^{h}+V_{\Gamma} e_{\Gamma}^{h}+w^{h} D^{2} b+\frac{1}{2} \nabla_{\Gamma} w^{h} \otimes \nabla_{\Gamma} w^{h}\right)\left(\nabla_{\Gamma} \hat{w} \otimes \nabla_{\Gamma} \bar{w}\right)\right] .
\end{aligned}
$$

Taking $\hat{\mathbf{e}}=\partial_{t} \overline{\mathbf{e}}=\partial_{t t} \mathbf{e}^{h}$, we obtain

$$
\begin{aligned}
& \left(\mathcal{M} \partial_{t t} \overline{\mathbf{e}}, \partial_{t} \overline{\mathbf{e}}\right)+\gamma \int_{\Gamma} \operatorname{tr}\left[\mathcal{C}\left(S_{\gamma} \bar{w}+G_{\Gamma} \bar{w}\right)\left(S_{\Gamma} \partial_{t} \bar{w}+G_{\Gamma} \partial_{t} \bar{w}\right)\right] \\
& \quad+\int_{\Gamma} \operatorname{tr}\left[\mathcal{C}\left(\varepsilon_{\Gamma} \overline{e_{\Gamma}}+V_{\Gamma} \overline{e_{\Gamma}}+\bar{w} D^{2} b+\frac{1}{2} \nabla_{\Gamma} w^{h} \otimes \nabla_{\Gamma} \bar{w}+\frac{1}{2} \nabla_{\Gamma} \bar{w} \otimes \nabla_{\Gamma} w^{h}\right)\right. \\
& \left.\quad \times\left(\varepsilon_{\Gamma} \partial_{t}-\overline{e_{\Gamma}}+V_{\Gamma} \partial_{t} e_{\Gamma}+\partial_{t} \bar{w} D^{2} b+\frac{1}{2} \nabla_{\Gamma} w^{h} \otimes \nabla_{\Gamma} \partial_{t} \bar{w}+\frac{1}{2} \nabla_{\Gamma} \partial_{t} \bar{w} \otimes \nabla_{\Gamma} w^{h}\right)\right] \\
& \quad+\int_{\Gamma} \operatorname{tr}\left[\mathcal{C}\left(\varepsilon_{\Gamma}\left(e_{\Gamma}\right)^{h}+V_{\Gamma} e_{\Gamma}^{h}+w^{h} D^{2} b+\frac{1}{2} \nabla_{\Gamma} w^{h} \otimes \nabla_{\Gamma} w^{h}\right)\left(\nabla_{\Gamma} \partial_{t} \bar{w} \otimes \nabla_{\Gamma} \bar{w}\right)\right]=0 .
\end{aligned}
$$

Since

$$
\begin{aligned}
\partial_{t} B_{M}\left(\mathbf{e}^{h}\right) & =\partial_{t}\left(\varepsilon_{\Gamma}\left(e_{\Gamma}\right)^{h}+V_{\Gamma} e_{\Gamma}^{h}+w^{h} D^{2} b+\frac{1}{2} \nabla_{\Gamma} w^{h} \otimes \nabla_{\Gamma} w^{h}\right) \\
& =\varepsilon_{\Gamma} \overline{e_{\Gamma}}+V_{\Gamma} \overline{e_{\Gamma}}+\bar{w} D^{2} b+\frac{1}{2} \nabla_{\Gamma} w^{h} \otimes \nabla_{\Gamma} \bar{w}+\frac{1}{2} \nabla_{\Gamma} \bar{w} \otimes \nabla_{\Gamma} w^{h}=B_{M}^{\prime}\left(\overline{\mathbf{e}}, \mathbf{e}^{h}\right)
\end{aligned}
$$

and $B_{F}(\overline{\mathbf{e}})=S_{\Gamma} \bar{w}+G_{\Gamma} \bar{w}$, it follows that we can rewrite (62) as 


$$
\begin{gathered}
\left(\mathcal{M} \partial_{t t} \overline{\mathbf{e}}, \partial_{t} \overline{\mathbf{e}}\right)+\int_{\Gamma}\left(\mathcal{C} B_{M}^{\prime}\left(\mathbf{e}^{h}, \overline{\mathbf{e}}\right)\right) \cdots \partial_{t} B_{M}^{\prime}\left(\mathbf{e}^{h}, \overline{\mathbf{e}}\right)+\gamma \int_{\Gamma}\left(\mathcal{C} B_{F}(\overline{\mathbf{e}})\right) \cdots \partial_{t} B_{F}(\overline{\mathbf{e}}) \\
\quad=\int_{\Gamma} \mathcal{C} B_{M}^{\prime}\left(\mathbf{e}^{h}, \overline{\mathbf{e}}\right) \cdots\left(\nabla_{\Gamma} \bar{w} \otimes \nabla_{\Gamma} \bar{w}\right)-\int_{\Gamma} \mathcal{C} B_{M}\left(\mathbf{e}^{h}\right) \cdots\left(\nabla_{\Gamma} \partial_{t} \bar{w} \otimes \nabla_{\Gamma} \bar{w}\right) .
\end{gathered}
$$

Let us define

$$
\bar{E}(t)=\left\|\mathcal{M} \partial_{t} \overline{\mathbf{e}}\right\|_{L^{2}(\Gamma)}+\int_{\Gamma} \mathcal{C} B_{M}^{\prime}\left(\mathbf{e}^{h}, \overline{\mathbf{e}}\right) \cdots B_{M}^{\prime}\left(\mathbf{e}^{h}, \overline{\mathbf{e}}\right)+\gamma \int_{\Gamma} \mathcal{C} B_{F}(\overline{\mathbf{e}}) \cdots B_{F}(\overline{\mathbf{e}}) .
$$

We have

$$
\left\|\mathcal{M} \partial_{t} \overline{\mathbf{e}}(t)\right\|_{L^{2}(\Gamma)}+\|\bar{w}(t)\|_{H^{2}}+\left\|\bar{e}_{\Gamma}(t)\right\|_{H^{1}} \leq \bar{E}(t) .
$$

It is straightforward to verify that $\bar{E}(t)$ is bounded above and below by a constant depending on $\|\overline{\mathbf{e}}\|_{V^{1}(\Gamma)}$ and $\left\|\partial_{t} \overline{\mathbf{e}}\right\|_{V^{0}(\Gamma)}$. This fact will be used frequently and without further mention. Eq. (64) can be rewritten as

$$
\begin{aligned}
\frac{1}{2} \partial_{t} \bar{E}(t)= & \int_{\Gamma} \mathcal{C} B_{M}^{\prime}\left(\mathbf{e}^{h}, \overline{\mathbf{e}}\right) \cdots\left(\nabla_{\Gamma} \bar{w} \otimes \nabla_{\Gamma} \bar{w}\right)-\int_{\Gamma} \mathcal{C} B_{M}\left(\mathbf{e}^{h}\right) \cdots\left(\nabla_{\Gamma} \partial_{t} \bar{w} \otimes \nabla_{\Gamma} \bar{w}\right) \\
= & \int_{\Gamma} \operatorname{tr}\left[\mathcal{C}\left(\varepsilon_{\Gamma} \bar{e}_{\Gamma}^{-}+V_{\Gamma} \bar{e}_{\Gamma}^{-}+\hat{w} D^{2} b+\frac{1}{2} \nabla_{\Gamma} w^{h} \otimes \nabla_{\Gamma} \bar{w}+\frac{1}{2} \nabla_{\Gamma} \bar{w} \otimes \nabla_{\Gamma} w^{h}\right)\left(\nabla_{\Gamma} \bar{w} \otimes \nabla_{\Gamma} \bar{w}\right)\right] \\
& -\int_{\Gamma} \operatorname{tr}\left[\mathcal{C}\left(\varepsilon_{\Gamma} e_{\Gamma}^{h}+V_{\Gamma} e_{\Gamma}^{h}+w^{h} D^{2} b+\frac{1}{2} \nabla_{\Gamma} w^{h} \otimes \frac{1}{2} \nabla_{\Gamma} w^{h}\right)\left(\nabla_{\Gamma} \partial_{t} \bar{w} \otimes \nabla_{\Gamma} \bar{w}\right)\right]
\end{aligned}
$$

Integrating this equation between 0 and $t$ yields

$$
\frac{1}{2}(\bar{E}(t)-\bar{E}(0))=\int_{0}^{t} \int_{\Gamma} \mathcal{C} B_{M}^{\prime}\left(\mathbf{e}^{h}, \overline{\mathbf{e}}\right) \cdots\left(\nabla_{\Gamma} \bar{w} \otimes \nabla_{\Gamma} \bar{w}\right)-\frac{1}{2} \int_{0}^{t} \int_{\Gamma} \mathcal{C} B_{M}\left(\mathbf{e}^{h}\right) \cdots \partial_{t}\left(\nabla_{\Gamma} \bar{w} \otimes \nabla_{\Gamma} \bar{w}\right) .
$$

Integrating by parts the second term of the right hand side of (68) gives

$$
\bar{E}(t)=\bar{E}(0)+3 \int_{0}^{t} \int_{\Gamma} \mathcal{C} B_{M}^{\prime}\left(\mathbf{e}^{h}, \overline{\mathbf{e}}\right) \cdots\left(\nabla_{\Gamma} \bar{w} \otimes \nabla_{\Gamma} \bar{w}\right)+\left[\int_{\Gamma}\left(\mathcal{C} B_{M}\left(\mathbf{e}^{h}\right)\right) \cdots\left(\nabla_{\Gamma} \bar{w} \otimes \nabla_{\Gamma} \bar{w}\right)\right]_{0}^{t} .
$$

Therefore

$$
\begin{aligned}
\bar{E}(t) \leq & \bar{E}(0)+\frac{3}{2} \int_{0}^{t}\left(\left\|\mathcal{C} B_{M}^{\prime}\left(\mathbf{e}^{h}, \overline{\mathbf{e}}\right)\right\|_{L^{2}(\Gamma)}^{2}+\left\|\nabla_{\Gamma} \bar{w} \otimes \nabla_{\Gamma} \bar{w}\right\|_{L^{2}(\Gamma)}^{2}\right) \mathrm{d} s \\
& +\left[\frac{1}{4 \epsilon}\left\|\mathcal{C} B_{M}\left(\mathbf{e}^{h}\right)\right\|_{L^{2}(\Gamma)}^{2}+\epsilon\left\|\nabla_{\Gamma} \bar{w} \otimes \nabla_{\Gamma} \bar{w}\right\|_{L^{2}(\Gamma)}^{2}\right]_{0}^{t}
\end{aligned}
$$

where $\epsilon$ is an arbitrarily small non-negative number.

From Lemma 4 we have $\left\|\nabla_{\Gamma} \bar{w} \otimes \nabla_{\Gamma} \bar{w}\right\|_{L^{2}(\Gamma)} \leq C\|\bar{w}\|_{H^{2}(\Gamma)}\|\bar{w}\|_{H^{1}(\Gamma)}$. Using (45) with (58) gives $\|\bar{w}\|_{H^{1}(\Gamma)} \leq$ $E_{h}(0)$, and then using (66), we obtain

$$
\|\nabla \bar{w} \otimes \nabla \bar{w}\|_{L^{2}(\Gamma)}^{2} \leq C E_{h}(0)\|\bar{w}\|_{H^{2}(\Gamma)}^{2} \leq C E_{h}(0) \bar{E}(t)
$$

where $C$ is a constant. In addition, the following inequalities follow from the fact that tensor $\mathcal{C}$ is coercive and the $a$ priori bound on the energies:

$$
\begin{aligned}
& \left\|\mathcal{C}\left(B_{M}\left(\mathbf{e}^{h}\right)\right)\right\|_{L^{2}(\Gamma)}^{2} \leq C E_{h}(t) \leq C E_{h}(0) \\
& \left\|\mathcal{C}\left(B_{M}^{\prime}\left(\mathbf{e}^{h}, \overline{\mathbf{e}}\right)\right)\right\|_{L^{2}(\Gamma)}^{2} \leq C \bar{E}(t)
\end{aligned}
$$

Therefore

$$
\bar{E}(t) \leq \bar{E}(0)+C\left(1+E_{h}(0)\right) \int_{0}^{t} \bar{E}(s) \mathrm{d} s+\frac{1}{4 \epsilon} C E_{h}(0)+C \epsilon E_{h}(0) \bar{E}(t) .
$$


Let us now set $\epsilon=1 /\left(2 C E_{h}(0)\right)$; then

$$
\bar{E}(t) \leq \bar{E}(0)+C\left(1+E_{h}(0)\right) \int_{0}^{t} \bar{E}(s) \mathrm{d} s+\frac{1}{2}\left(C E_{h}(0)\right)^{2}+\frac{1}{2} \bar{E}(t)
$$

and therefore

$$
\bar{E}(t) \leq C\left(\bar{E}(0)+\left(E_{h}(0)\right)^{2}\right)+C\left(1+E_{h}(0)\right) \int_{0}^{t} \bar{E}(s) \mathrm{d} s .
$$

Gronwall's lemma yields

$$
\forall t \in\left[0, \tau\left[, \quad \bar{E}(t) \leq C\left(\bar{E}(0)+\left[E_{h}(0)\right]^{2}\right) \exp \left(C\left(1+E_{h}(0)\right) \tau\right)\right.\right.
$$

where $C$ does not depend on $h$.

\subsection{A priori bounds for the discrete initial data}

In order to provide an effective estimate (independent of $h$ ) of the right hand side of Eq. (76) we need to estimate $E_{h}(0)$ and $\bar{E}(0)$. This is the point of the next two sections.

\subsubsection{A priori bounds for $E_{h}(0)$}

We consider $\mathbf{e}^{0, h}$ and $\mathbf{e}^{1, h}$ "good" approximations of the initial data, that is

$$
\lim _{h \rightarrow 0}\left\|\mathbf{e}^{0}-\mathbf{e}^{h, 0}\right\|_{V^{2}(\Gamma)}=0 \quad \text { and } \quad \lim _{h \rightarrow 0}\left\|\mathbf{e}^{1}-\mathbf{e}^{h, 1}\right\|_{V^{1}(\Gamma)}=0 .
$$

By the stability result of the estimates resulting from (77) and the regularity of the initial condition, we obtain $E_{h}(0) \leq C_{2}$.

\subsubsection{A priori bounds for $\bar{E}(0)$}

Eq. (57a) along with the coercivity of $\mathcal{M}$ gives

$$
\left(\partial_{t} \overline{\mathbf{e}}(0), \hat{\mathbf{e}}\right)_{V^{0}(\Gamma)} \leq C\left|\int_{\Gamma}\left(B_{M}\left(\mathbf{e}^{h, 0}\right)\right) \cdots B_{M}^{\prime}\left(\mathbf{e}^{h, 0}, \hat{\mathbf{e}}\right)\right|+C\left|\int_{\Gamma}\left(B_{F}\left(\mathbf{e}^{h, 0}\right)\right) \cdots B_{F}(\hat{\mathbf{e}})\right| .
$$

It follows that

$$
\begin{aligned}
\left(\partial_{t} \overline{\mathbf{e}}(0), \hat{\mathbf{e}}\right)_{V^{0}(\Gamma)} \leq & C\left|\int_{\Gamma} \operatorname{div}_{\Gamma} B_{M}\left(\mathbf{e}^{h, 0}\right) \cdots \hat{e}_{\Gamma}\right|+C\left|\int_{\Gamma} B_{M}\left(\mathbf{e}^{h, 0}\right) \cdots\left(V_{\Gamma} \hat{e_{\Gamma}}+\hat{w} D^{2} b\right)\right| \\
& +C\left|\int_{\Gamma}\left\langle\left(\varepsilon_{\Gamma} e_{\Gamma}^{h, 0}+V_{\Gamma} e_{\Gamma}^{h, 0}+w^{h, 0} D^{2} b\right) \nabla_{\Gamma} w^{h, 0}, \nabla_{\Gamma} \hat{w}\right\rangle\right| \\
& +C\left|\int_{\Gamma}\left\langle\left(\nabla_{\Gamma} w^{h, 0} \otimes \nabla_{\Gamma} w^{h, 0}\right) \nabla_{\Gamma} w^{h, 0}, \nabla_{\Gamma} \hat{w}\right\rangle\right| \\
& +C\left|\int_{\Gamma} S_{\Gamma} w^{h, 0} \cdots S_{\Gamma} \hat{w}\right|+C\left|\int_{\Gamma} G_{\Gamma} w^{h, 0} \cdots S_{\Gamma} \hat{w}\right| \\
& +C\left|\int_{\Gamma}\left(S_{\Gamma} w^{h, 0}+G_{\Gamma} w^{h, 0}\right) \cdots G_{\Gamma} \hat{w}\right|
\end{aligned}
$$

and therefore

$$
\begin{aligned}
\left(\partial_{t} \overline{\mathbf{e}}(0), \hat{\mathbf{e}}\right)_{V^{0}(\Gamma)} \leq & C\left\|\operatorname{div}_{\Gamma} B_{M}\left(\mathbf{e}^{h, 0}\right)\right\|_{L^{2}(\Gamma)}\left\|\hat{e_{\Gamma}}\right\|_{L^{2}(\Gamma)}+C\left\|B_{M}\left(\mathbf{e}^{h, 0}\right)\right\|_{L^{2}(\Gamma)}\left\|V_{\Gamma} \hat{e_{\Gamma}}+\hat{w} D^{2} b\right\|_{L^{2}(\Gamma)} \\
& +C\left\|\left(\varepsilon_{\Gamma} e_{\Gamma}^{h, 0}+V_{\Gamma} e_{\Gamma}^{h, 0}+w^{h, 0} D^{2} b\right)\right\|_{L^{4}(\Gamma)}\left\|\nabla_{\Gamma} w^{h, 0}\right\|_{L^{4}(\Gamma)}\|\hat{w}\|_{H^{1}(\Gamma)} \\
& +C\left\|\nabla_{\Gamma} w^{h, 0}\right\|_{L^{6}(\Gamma)}^{3}\|\hat{w}\|_{H^{1}(\Gamma)} \\
& +C\left|\int_{\Gamma}\left\langle\operatorname{div}_{\Gamma} S_{\Gamma} w^{h, 0}, \nabla_{\Gamma} \hat{w}\right\rangle\right|+C\left|\int_{\Gamma}\left\langle\operatorname{div}_{\Gamma} G_{\Gamma} w^{h, 0}, \nabla_{\Gamma} \hat{w}\right\rangle\right| \\
& +C\left\|w^{h, 0}\right\|_{H^{2}(\Gamma)}\|\hat{w}\|_{H^{1}(\Gamma)} .
\end{aligned}
$$


Using $H^{1}(\Gamma) \subset L^{6}(\Gamma) \subset L^{4}(\Gamma)$ as well as $\|\hat{w}\|_{H^{1}(\Gamma)} \leq\|\hat{\mathbf{e}}\|_{V^{0}(\Gamma)}$ and $\left\|\hat{e}_{\Gamma}\right\|_{L^{2}(\Gamma)} \leq\|\hat{\mathbf{e}}\|_{V^{0}(\Gamma)}$ we get

$$
\begin{aligned}
\left(\partial_{t} \overline{\mathbf{e}}(0), \hat{\mathbf{e}}\right)_{V^{0}(\Gamma)} & \leq C\left(\left\|\mathbf{e}^{h, 0}\right\|_{V^{0}(\Gamma)}+\left\|\mathbf{e}^{h, 0}\right\|_{V^{1}(\Gamma)}+\left\|\mathbf{e}^{h, 0}\right\|_{V^{2}(\Gamma)}\right)\|\hat{\mathbf{e}}\|_{V^{0}(\Gamma)} \\
& \leq C\left\|\mathbf{e}^{h, 0}\right\|_{V^{2}(\Gamma)}\|\hat{\mathbf{e}}\|_{V^{0}(\Gamma)} .
\end{aligned}
$$

For $h$ small enough, $\left\|\mathbf{e}^{h, 0}\right\|_{V^{2}(\Gamma)} \leq 2\left\|\mathbf{e}^{0}\right\|_{V^{2}(\Gamma)}$, and it follows that

$$
\left(\partial_{t} \overline{\mathbf{e}}(0), \hat{\mathbf{e}}\right)_{V^{0}(\Gamma)} \leq C\left\|\mathbf{e}^{0}\right\|_{V^{2}(\Gamma)}\|\hat{\mathbf{e}}\|_{V^{0}(\Gamma)}
$$

where $C$ is a constant independent of $h$; therefore

$$
\left\|\partial_{t} \overline{\mathbf{e}}(0)\right\|_{V^{0}(\Gamma)} \leq C\left\|\mathbf{e}^{0}\right\|_{V^{2}(\Gamma)} .
$$

Using $\|\overline{\mathbf{e}}(0)\|_{V^{2}(\Gamma)} \leq C$ and (83) we conclude that $\bar{E}(0)$ is bounded by a constant independent of $h$. Eq. (76) yields, for all $t<\tau$,

$$
\bar{E}(t) \leq C .
$$

Hence the a priori bounds

$$
\left\|\mathbf{e}^{h}\right\|_{V^{1}(\Gamma)}+\left\|\partial_{t} \mathbf{e}^{h}\right\|_{V^{1}(\Gamma)}+\left\|\partial_{t t} \mathbf{e}^{h}\right\|_{V^{0}(\Gamma)} \leq C
$$

hold uniformly in $h$, for all $t<\tau$ where $\tau$ is arbitrary.

\subsection{Passage to the limit}

We can now extract convergent subsequences, denoted by the same symbol, such that the following weak* convergence holds:

$$
\begin{aligned}
& \mathbf{e}^{h} \rightarrow \mathbf{e} \quad \text { in } L^{\infty}\left(0, \tau ; V^{1}(\Gamma)\right) \\
& \partial_{t} \mathbf{e}^{h} \rightarrow \partial_{t} \mathbf{e} \quad \text { in } L^{\infty}\left(0, \tau ; V^{1}(\Gamma)\right) \\
& \partial_{t t} \mathbf{e}^{h} \rightarrow \partial_{t t} \mathbf{e} \quad \text { in } L^{\infty}\left(0, \tau ; V^{0}(\Gamma)\right) .
\end{aligned}
$$

Now, we can pass to the limit on the original semidiscrete form of Eq. (57a). Note that this is possible due to the weak continuity of $\nabla_{\Gamma} w \otimes \nabla_{\Gamma} w$. The clamped boundary condition also passes to the limit thanks to the strong convergence of the boundary traces. Therefore, we can conclude that e satisfies the weak form of the original equation (16). Moreover, it has the following regularity

$$
\mathbf{e} \in C\left(0, \tau, V^{1}(\Gamma)\right), \quad \partial_{t} \mathbf{e} \in C\left(0, \tau, V^{1}(\Gamma)\right), \quad \partial_{t t} \mathbf{e} \in C\left(0, \tau, V^{0}(\Gamma)\right) .
$$

In order to obtain existence of weak solutions, we use the a priori bound for the original energy function which implies weak convergence of

$$
\begin{aligned}
& \mathbf{e}^{h} \rightarrow \mathbf{e} \quad \text { in } L^{\infty}\left(0, \tau ; V^{1}(\Gamma)\right) \\
& \partial_{t} \mathbf{e}^{h} \rightarrow \partial_{t} \mathbf{e} \quad \text { in } L^{\infty}\left(0, \tau ; V^{0}(\Gamma)\right) .
\end{aligned}
$$

The above weak convergence and weak continuity of nonlinear terms allows passing to the limit. This part of the argument is straightforward. The limit equation defines a weak solution.

\subsection{The improved spatial regularity}

Using the second equation of (12) we have

$$
\begin{aligned}
& \operatorname{div}_{\Gamma}\left(\mathcal{C}\left(\varepsilon_{\Gamma} e_{\Gamma}+V_{\Gamma} e_{\Gamma}\right)\right) \\
& \quad=\rho\left(I+\gamma\left(D^{2} b\right)^{2}\right) \partial_{t t} e_{\Gamma}-\rho \frac{\gamma}{2} D^{2} b \partial_{t t} w-\operatorname{div}_{\Gamma}\left(\mathcal{C}\left(w D^{2} b\right)\right)-\frac{1}{2} \operatorname{div}_{\Gamma}\left(\mathcal{C}\left(\nabla_{\Gamma} w \otimes \nabla_{\Gamma} w\right)\right) .
\end{aligned}
$$


The regularity established in Section 6.4 gives the following regularities:

$$
\partial_{t t} e_{\Gamma} \in\left[L^{2}(\Gamma)\right]^{2}, \quad \partial_{t t} e_{\Gamma} \in\left[L^{2}(\Gamma)\right]^{2}, \quad \operatorname{div}_{\Gamma}\left(\mathcal{C}\left(w D^{2} b\right)\right) \in\left[H^{1}(\Gamma)\right]^{2} .
$$

In addition $\nabla_{\Gamma} w \otimes \nabla_{\Gamma} w$ is in $\left[H^{1-\epsilon}(\Gamma)\right]^{2}$ as the product of two functions in $\left[H^{1}(\Gamma)\right]^{2}$. It follows that

$$
\operatorname{div}_{\Gamma}\left(\mathcal{C}\left(\nabla_{\Gamma} w \otimes \nabla_{\Gamma} w\right)\right) \in\left[H^{-\epsilon}(\Gamma)\right]^{2}
$$

and consequently $\operatorname{div}_{\Gamma}\left(\mathcal{C}\left(\varepsilon_{\Gamma} e_{\Gamma}+V_{\Gamma} e_{\Gamma}\right)\right) \in\left[H^{-\epsilon}(\Gamma)\right]^{2}$ so

$$
e_{\Gamma} \in C\left(0, \tau ;\left[H^{2-\epsilon}(\Gamma)\right]^{2}\right) .
$$

For $w$, the first equation of (12) gives

$$
\begin{aligned}
(\lambda+ & 2 \mu) \gamma \Delta_{\Gamma}^{2} w=-\rho \partial_{t t} w+\rho \gamma \Delta_{\Gamma} \partial_{t t} w-\rho \frac{\gamma}{2} \operatorname{div}_{\Gamma}\left(D^{2} b \partial_{t t} e_{\Gamma}\right)-\operatorname{tr}\left(\mathcal{C}\left(\varepsilon_{\Gamma} e_{\Gamma}+V_{\Gamma} e_{\Gamma}\right) D^{2} b\right) \\
& -\operatorname{tr}\left(\mathcal{C}\left(w D^{2} b+\frac{1}{2} \nabla_{\Gamma} w \otimes \nabla_{\Gamma} w\right) D^{2} b\right)+\operatorname{div}_{\Gamma}\left(\mathcal{C}\left(\varepsilon_{\Gamma} e_{\Gamma}+V_{\Gamma} e_{\Gamma}\right) \nabla_{\Gamma} w\right) \\
& +\operatorname{div}_{\Gamma}\left(\mathcal{C}\left(w D^{2} b+\frac{1}{2} \nabla_{\Gamma} w \otimes \nabla_{\Gamma} w\right) \nabla_{\Gamma} w\right)-2 \mu \gamma \operatorname{div}_{\Gamma}\left(K \nabla_{\Gamma} w\right)-2 \mu \gamma \operatorname{div}_{\Gamma}\left(\left(D^{2} b\right)^{2} \nabla w\right) .
\end{aligned}
$$

We now inspect the regularity of each term of (92). This regularity follows from Section 6.4 for all but the fifth term:

$$
\begin{aligned}
& \partial_{t t} w \in H^{1}(\Gamma), \quad \Delta_{\Gamma} \partial_{t t} w \in H^{-1}(\Gamma), \quad \operatorname{div}_{\Gamma}\left(D^{2} b \partial_{t t} e_{\Gamma}\right) \in H^{-1}(\Gamma) \\
& \operatorname{tr}\left(\mathcal{C}\left(\varepsilon_{\Gamma} e_{\Gamma}+V_{\Gamma} e_{\Gamma}\right) D^{2} b\right) \in L^{2}(\Gamma), \quad \operatorname{tr}\left(\mathcal{C}\left(w D^{2} b+\frac{1}{2} \nabla_{\Gamma} w \otimes \nabla_{\Gamma} w\right) D^{2} b\right) \in H^{1-\epsilon}(\Gamma) \\
& \operatorname{div}_{\Gamma}\left(\mathcal{C}\left(w D^{2} b+\frac{1}{2} \nabla_{\Gamma} w \otimes \nabla_{\Gamma} w\right) \nabla_{\Gamma} w\right) \in H^{-\epsilon}(\Gamma), \quad \operatorname{div}_{\Gamma}\left(K \nabla_{\Gamma} w\right) \in L^{2}(\Gamma), \\
& \operatorname{div}_{\Gamma}\left(\left(D^{2} b\right)^{2} \nabla w\right) \in L^{2}(\Gamma)
\end{aligned}
$$

where we have used that $\nabla w \in\left[H^{1}(\Gamma)\right]^{2}$ implies $\nabla_{\Gamma} w \otimes \nabla_{\Gamma} w \in\left[H^{1-\epsilon}(\Gamma)\right]^{2}$. The regularity of the sixth term of (92) follows from (91). We have $\mathcal{C}\left(\varepsilon_{\Gamma} e_{\Gamma}+V_{\Gamma} e_{\Gamma}\right) \in H^{1-\epsilon}(\Gamma)$ and $\nabla_{\Gamma} w \in\left[H^{1}(\Gamma)\right]^{2}$ so the product is in $H^{1-\epsilon}(\Gamma)$,

$$
\operatorname{div}_{\Gamma}\left(\mathcal{C}\left(\varepsilon_{\Gamma} e_{\Gamma}+V_{\Gamma} e_{\Gamma}\right) \nabla_{\Gamma} w\right) \in\left[H^{-\epsilon}(\Gamma)\right]^{2}
$$

and consequently $\Delta_{\Gamma}^{2} w$ belongs to $H^{-1}(\Gamma)$ so

$$
w \in C\left(0, \tau ; H^{3}(\Gamma)\right) .
$$

Going back to (90) we further improve the regularity with $\epsilon$, obtaining $e_{\Gamma} \in C\left(0, \tau ;\left[H^{2}(\Gamma)\right]^{2}\right)$. It follows that

$$
\mathbf{e} \in C\left(0, \tau, V^{2}(\Gamma)\right)
$$

as desired for the proof of the existence of regular solutions.

\section{Proof of Theorem 2 (uniqueness property)}

In order to prove Theorem 2 we use a method developed by V.I. Sedenko for Marguerre-Vlasov equations and then utilized for the von Kármán equations in [25]. Let $\mathbf{e}^{1}$ and $\mathbf{e}^{2}$ be two weak solutions to the small finite deflections shell problem, with the same initial condition. Let $\tilde{\mathbf{e}}=\mathbf{e}^{2}-\mathbf{e}^{1}$. The goal of this section is to prove that $\tilde{\mathbf{e}}=0$.

\subsection{First step}

Lemma 7. The following inequality holds true

$$
\left\|\mathbb{A}^{-1}\left(\begin{array}{c}
\tilde{\mathbf{e}} \\
\partial_{t} \tilde{\mathbf{e}}
\end{array}\right)\right\|_{\mathcal{H}} \leq \int_{0}^{t}\left\|\mathbb{A}^{-1}\left(\begin{array}{c}
0 \\
\mathcal{M}^{-1}\left[N\left(\mathbf{e}^{2}\right)-N\left(\mathbf{e}^{1}\right)\right]
\end{array}\right)\right\|_{\mathcal{H}} \mathrm{d} s .
$$


Proof. Eq. (20) yields

$$
\partial_{t} \mathbb{A}^{-1}\left(\begin{array}{c}
\tilde{\mathbf{e}} \\
\partial_{t} \tilde{\mathbf{e}}
\end{array}\right)+\mathbb{A}^{-1}\left(\begin{array}{c}
\tilde{\mathbf{e}} \\
\partial_{t} \tilde{\mathbf{e}}
\end{array}\right)=\mathbb{A}^{-1}\left(\begin{array}{c}
0 \\
\mathcal{M}^{-1}\left[N\left(\mathbf{e}^{2}\right)-N\left(\mathbf{e}^{1}\right)\right]
\end{array}\right)
$$

and consequently

$$
\begin{gathered}
\left(\partial_{t} \mathbb{A}^{-1}\left(\begin{array}{c}
\tilde{\mathbf{e}} \\
\partial_{t} \tilde{\mathbf{e}}
\end{array}\right), \mathbb{A}^{-1}\left(\begin{array}{c}
\tilde{\mathbf{e}} \\
\partial_{t} \tilde{\mathbf{e}}
\end{array}\right)\right)_{\mathcal{H}}+\left(\mathbb{A}^{-1}\left(\begin{array}{c}
\tilde{\mathbf{e}} \\
\partial_{t} \tilde{\mathbf{e}}
\end{array}\right), \mathbb{A}^{-1}\left(\begin{array}{c}
\tilde{\mathbf{e}} \\
\partial_{t} \tilde{\mathbf{e}}
\end{array}\right)\right)_{\mathcal{H}} \\
=\left(\mathbb{A}^{-1}\left(\begin{array}{c}
0 \\
\mathcal{M}_{\gamma}^{-1}\left[N\left(\mathbf{e}^{2}\right)-N\left(\mathbf{e}^{1}\right)\right]
\end{array}\right), \mathbb{A}^{-1}\left(\begin{array}{c}
\tilde{\mathbf{e}} \\
\partial_{t} \tilde{\mathbf{e}}
\end{array}\right)\right)_{\mathcal{H}} .
\end{gathered}
$$

Within this proof, we shall define

$$
X(s)=\mathbb{A}^{-1}\left(\begin{array}{c}
\tilde{\mathbf{e}}(s) \\
\partial_{t} \tilde{\mathbf{e}}(s)
\end{array}\right) \quad \text { and } \quad F(s)=\mathbb{A}^{-1}\left(\begin{array}{c}
0 \\
\mathcal{M}^{-1}\left[N\left(\mathbf{e}^{2}\right)-N\left(\mathbf{e}^{1}\right)\right]
\end{array}\right) .
$$

Using Lemma 2 on (96) yields

$$
\left(\partial_{t} X(s), X(s)\right)_{\mathcal{H}}=(X(s), F(s))_{\mathcal{H}}
$$

and therefore

$$
\frac{1}{2} \int_{0}^{t} \partial_{s}\|X(s)\|_{\mathcal{H}}^{2} \mathrm{~d} s=\int_{0}^{t}(X(s), F(s))_{\mathcal{H}} \mathrm{d} s
$$

whence it follows that

$$
\frac{1}{2}\|X(t)\|_{\mathcal{H}}^{2} \leq \frac{1}{2}\|X(0)\|_{\mathcal{H}}^{2}+\int_{0}^{t}\|X(s)\|_{\mathcal{H}}\|F(s)\|_{\mathcal{H}} \mathrm{d} s .
$$

Since $\tilde{\mathbf{e}}(0)=\partial_{t} \mathbf{e}(0)=0$ and $\mathbb{A}^{-1}$ is linear, we have $X(0)=0$; it follows that

$$
\frac{1}{2}\|X(t)\|_{\mathcal{H}}^{2} \leq\left(\sup _{s \in[0, t]}\|X(s)\|_{\mathcal{H}}\right)\left(\int_{0}^{t}\|F(s)\|_{\mathcal{H}} \mathrm{d} s\right)
$$

and thus, for all $\bar{t} \leq t$, we have

$$
\left\{\begin{array}{l}
\frac{1}{2}\|X(\bar{t})\|_{\mathcal{H}}^{2} \leq\left(\sup _{s \in[0, \bar{t}]}\|X(s)\|_{\mathcal{H}}\right)\left(\int_{0}^{\bar{t}}\|F(s)\|_{\mathcal{H}} \mathrm{d} s\right) \\
\sup _{s \in[0, \bar{t}]}\|X(s)\|_{\mathcal{H}}^{2} \leq \sup _{s \in[0, t]}\|X(s)\|_{\mathcal{H}}^{2} \\
\int_{0}^{\bar{t}}\|F(s)\|_{\mathcal{H}} \mathrm{d} s \leq \int_{0}^{t}\|F(s)\|_{\mathcal{H}} \mathrm{d} s
\end{array}\right.
$$

whence it follows that

$$
\frac{1}{2}\|X(\bar{t})\|_{\mathcal{H}}^{2} \leq\left(\sup _{s \in[0, t]}\|X(s)\|_{\mathcal{H}}\right)\left(\int_{0}^{t}\|F(s)\|_{\mathcal{H}} \mathrm{d} s\right)
$$

and thus

$$
\frac{1}{2} \sup _{\bar{t} \in[0, t]}\|X(\bar{t})\|_{\mathcal{H}}^{2} \leq\left(\sup _{s \in[0, t]}\|X(s)\|_{\mathcal{H}}^{2}\right)\left(\int_{0}^{t}\|F(s)\|_{\mathcal{H}} \mathrm{d} s\right)
$$

and so consequently

$$
\sup _{s \in[0, t]}\|X(s)\|_{\mathcal{H}} \leq \int_{0}^{t}\|F(s)\|_{\mathcal{H}} \mathrm{d} s
$$


and using $\|X(t)\|_{\mathcal{H}} \leq \sup _{s \in[0, t]}\|X(s)\|_{\mathcal{H}}$, we get

$$
\|X(t)\|_{\mathcal{H}} \leq \int_{0}^{t}\|F(s)\|_{\mathcal{H}} \mathrm{d} s,
$$

that is (95).

Lemma 8. The following inequality holds true

$$
\left\|\mathcal{M}_{\gamma}^{1 / 2} \tilde{\mathbf{e}}\right\|_{L^{2}} \leq\left\|\mathbb{A}^{-1}\left(\begin{array}{c}
\tilde{\mathbf{e}} \\
\partial_{t} \tilde{\mathbf{e}}
\end{array}\right)\right\|_{\mathcal{H}} .
$$

Proof. We have

$$
\mathbb{A}^{-1}\left(\begin{array}{c}
\mathbf{e} \\
\partial_{t} \tilde{\mathbf{e}}
\end{array}\right)=\left(\begin{array}{c}
\mathcal{A}^{-1} \mathcal{M}_{\gamma} \partial_{t} \mathbf{e} \\
-\tilde{\mathbf{e}}
\end{array}\right)
$$

and therefore

$$
\left\|\mathbb{A}^{-1}\left(\begin{array}{c}
\tilde{\mathbf{e}} \\
\partial_{t} \mathbf{e}
\end{array}\right)\right\|_{\mathcal{H}}^{2}=\left\|\mathcal{A}^{-1 / 2} \mathcal{M}_{\gamma} \partial_{t} \tilde{\mathbf{e}}\right\|_{L^{2}}^{2}+\left\|\mathcal{M}_{\gamma}^{1 / 2} \tilde{\mathbf{e}}\right\|_{L^{2}}^{2} .
$$

Inequality (97) follows.

Proposition 3. The following inequality holds true

$$
\left\|\mathcal{M}_{\gamma}^{1 / 2} \tilde{\mathbf{e}}\right\|_{L^{2}} \leq \int_{0}^{t}\left\|\mathcal{A}^{-1 / 2}\left[N\left(\mathbf{e}^{2}(s)\right)-N\left(\mathbf{e}^{1}\right)(s)\right]\right\|_{L^{2}} \mathrm{~d} s .
$$

Proof. We have

$$
\mathbb{A}^{-1}\left(\begin{array}{c}
0 \\
\mathcal{M}_{\gamma}^{-1}\left[N\left(\mathbf{e}^{2}\right)-N\left(\mathbf{e}^{1}\right)\right]
\end{array}\right)=\left(\begin{array}{c}
\mathcal{A}^{-1}\left[N\left(\mathbf{e}^{2}\right)-N\left(\mathbf{e}^{1}\right)\right] \\
0
\end{array}\right)
$$

and therefore

$$
\left\|\mathbb{A}^{-1}\left(\begin{array}{c}
0 \\
\mathcal{M}_{\gamma}^{-1}\left[N\left(\mathbf{e}^{2}\right)-N\left(\mathbf{e}^{1}\right)\right]
\end{array}\right)\right\|_{\mathcal{H}}=\left\|\mathcal{A}^{-1 / 2}\left[N\left(\mathbf{e}^{2}\right)-N\left(\mathbf{e}^{1}\right)\right]\right\|_{L^{2}} .
$$

From Lemma 7, we get

$$
\left\|\mathbb{A}^{-1}\left(\begin{array}{c}
\mathbf{e} \\
\partial_{t} \mathbf{e}
\end{array}\right)\right\|_{\mathcal{H}} \leq \int_{0}^{t}\left\|\mathcal{A}^{-1 / 2}\left[N\left(\mathbf{e}^{2}\right)-N\left(\mathbf{e}^{1}\right)\right]\right\|_{L^{2}} \mathrm{~d} s .
$$

Lemma 8 yields

$$
\left\|\mathcal{M}_{\gamma}^{1 / 2} \tilde{\mathbf{e}}\right\|_{L^{2}} \leq \int_{0}^{t}\left\|\mathcal{A}^{-1 / 2}\left[N\left(\mathbf{e}^{2}\right)-N\left(\mathbf{e}^{1}\right)\right]\right\|_{L^{2}} \mathrm{~d} s
$$

which proves (98).

Corollary 2. Following Proposition 3, we obtain

$$
\left\|\tilde{e}_{\Gamma}\right\|_{L^{2}}^{2}+\|\tilde{w}\|_{H^{1}}^{2} \leq t \int_{0}^{t}\left(\left\|N_{\Gamma}\left(w^{2}\right)-N_{\Gamma}\left(w^{1}\right)\right\|_{H^{-1}}^{2}+\left\|N_{n}\left(e_{\Gamma}^{2}, w^{2}\right)-N_{n}\left(e_{\Gamma}^{1}, w^{1}\right)\right\|_{H^{-2}}^{2}\right) \mathrm{d} s .
$$

Proof. The corollary follows from the use of $\left(\int_{0}^{t} f(t) \mathrm{d} t\right)^{2} \leq t \int_{0}^{t} f(t)^{2} \mathrm{~d} t$ on (98). 


\subsection{Second step}

Lemma 9. We have

$$
\begin{aligned}
& N_{\Gamma}\left(w^{2}\right)-N_{\Gamma}\left(w^{1}\right)=\lambda \nabla_{\Gamma}\left(\left\langle\nabla_{\Gamma} \tilde{w}, \nabla_{\Gamma}\left(w^{2}+w^{1}\right)\right\rangle\right) \\
& \quad+2 \mu \operatorname{div}_{\Gamma}\left(\nabla_{\Gamma} \tilde{w} \otimes \nabla_{\Gamma} w^{2}+\nabla_{\Gamma} w^{1} \otimes \nabla_{\Gamma} \tilde{w}\right) \\
& N_{n}\left(e_{\Gamma}^{2}, w^{2}\right)-N_{n}\left(e_{\Gamma}^{1}, w^{1}\right)=-2 H \lambda\left\langle\nabla_{\Gamma} \tilde{w}, \nabla_{\Gamma}\left(w^{2}+w^{1}\right)\right\rangle \\
& \quad+4 \lambda \operatorname{div}_{\Gamma}\left(H w^{2} \nabla_{\Gamma} \tilde{w}+H \tilde{w} \nabla_{\Gamma} w^{1}\right)+4 \mu \operatorname{div}_{\Gamma}\left(D^{2} b\left(w^{2} \nabla_{\Gamma} \tilde{w}+\tilde{w} \nabla_{\Gamma} w^{1}\right)\right) \\
& \quad+(\lambda+2 \mu) \operatorname{div}_{\Gamma}\left(\left\|\nabla_{\Gamma} w^{2}\right\|^{2} \nabla_{\Gamma} \tilde{w}+\nabla_{\Gamma} w^{1}\left\langle\nabla_{\Gamma} \tilde{w}, \nabla_{\Gamma}\left(w^{2}+w^{1}\right)\right\rangle\right) \\
& \quad-2 \mu\left\langle D^{2} b \nabla_{\Gamma} \tilde{w}, \nabla_{\Gamma} w^{2}\right\rangle-2 \mu\left\langle D^{2} b \nabla_{\Gamma} w^{1}, \nabla_{\Gamma} \tilde{w}\right\rangle-2 \lambda \operatorname{div}_{\Gamma}\left(\operatorname{div}_{\Gamma} \tilde{e}_{\Gamma} \nabla_{\Gamma} w^{2}+\operatorname{div}_{\Gamma} e_{\Gamma}^{1} \nabla_{\Gamma} \tilde{w}\right) \\
& \quad-4 \mu \operatorname{div}_{\Gamma}\left(\left(\varepsilon_{\Gamma} e_{\Gamma}^{2}+V_{\Gamma} e_{\Gamma}^{2}\right) \nabla_{\Gamma} \tilde{w}+\left(\varepsilon_{\Gamma} \tilde{e}_{\Gamma}+V_{\Gamma} \tilde{e}_{\Gamma}\right) \nabla_{\Gamma} w^{1}\right) .
\end{aligned}
$$

Proposition 4. Let $\epsilon>0$ and $\alpha>0$ be such that $\epsilon+\alpha \leq 1$. There exists a real C depending only on $\lambda, \mu, w^{1}$ and $w^{2}$ such that

$$
\left\|N_{\Gamma}\left(w^{2}\right)-N_{\Gamma}\left(w^{1}\right)\right\|_{H^{-1}}^{2} \leq C \ln \left(1+\lambda_{n}\right)\|\tilde{w}\|_{H^{1}}^{2}+\frac{C}{\lambda_{n}^{\alpha}}
$$

Proof. Following Definition 7 gives

$$
\begin{aligned}
\left\|N_{\Gamma}\left(w^{2}\right)-N_{\Gamma}\left(w^{1}\right)\right\|_{H^{-1}}^{2} \leq & \lambda\left\|\nabla_{\Gamma}\left\langle\nabla_{\Gamma} \tilde{w}, \nabla_{\Gamma}\left(w^{2}+w^{1}\right)\right\rangle\right\|_{H^{-1}}^{2} \\
& +2 \mu\left\|\operatorname{div}_{\Gamma}\left(\nabla_{\Gamma} \tilde{w} \otimes \nabla_{\Gamma} w^{2}+\nabla_{\Gamma} w^{1} \otimes \nabla_{\Gamma} \tilde{w}\right)\right\|_{H^{-1}}^{2} .
\end{aligned}
$$

Since $\operatorname{div}_{\Gamma}$ is a bounded operator on $H^{-1}$, there exists a constant $C$ such that

$$
\begin{aligned}
\left\|N_{\Gamma}\left(w^{2}\right)-N_{\Gamma}\left(w^{1}\right)\right\|_{H^{-1}}^{2} \leq & \lambda^{2} C\left\|\left\langle\nabla_{\Gamma} \tilde{w}, \nabla_{\Gamma}\left(w^{2}+w^{1}\right)\right\rangle\right\|_{L^{2}}^{2} \\
& +4 \mu^{2} C\left\|\nabla_{\Gamma} \tilde{w} \otimes \nabla_{\Gamma} w^{2}+\nabla_{\Gamma} w^{1} \otimes \nabla_{\Gamma} \tilde{w}\right\|_{L^{2}}^{2} .
\end{aligned}
$$

Applying (55) with $v_{1}=\nabla_{\Gamma} \tilde{w}$ and $v_{2}=\nabla_{\Gamma}\left(w^{2}+w^{1}\right)$ yields

$$
\left\|\left\langle\nabla_{\Gamma} \tilde{w}, \nabla_{\Gamma}\left(w^{2}+w^{1}\right)\right\rangle\right\|_{L^{2}}^{2} \leq C\left(\ln \left(1+\lambda_{n}\right)\|\tilde{w}\|_{H^{1}}^{2}+\frac{1}{\lambda_{n}^{\alpha}}\left\|w^{2}-w^{1}\right\|_{H^{1+\epsilon+\alpha}}^{2}\right)\left\|w^{2}+w^{1}\right\|_{H^{2}}^{2} .
$$

Applying (56) with $v_{1}=\nabla_{\Gamma} \tilde{w}$ and $v_{2}=\nabla_{\Gamma} w^{2}$ yields

$$
\left\|\nabla_{\Gamma} \tilde{w} \otimes \nabla_{\Gamma} w^{2}\right\|_{L^{2}}^{2} \leq C\left(\ln \left(1+\lambda_{n}\right)\|\tilde{w}\|_{H^{1}}^{2}+\frac{1}{\lambda_{n}^{\alpha}}\left\|w^{2}-w^{1}\right\|_{H^{1+\epsilon+\alpha}}^{2}\right)\left\|w^{2}\right\|_{H^{2}}^{2} .
$$

Applying (56) with $v_{1}=\nabla_{\Gamma} \tilde{w}$ and $v_{2}=\nabla_{\Gamma} w^{1}$ yields

$$
\left\|\nabla_{\Gamma} w^{1} \otimes \nabla_{\Gamma} \tilde{w}\right\|_{L^{2}} \leq C\left(\ln \left(1+\lambda_{n}\right)\|\tilde{w}\|_{H^{1}}^{2}+\frac{1}{\lambda_{n}^{\alpha}}\left\|w^{2}-w^{1}\right\|_{H^{1+\epsilon+\alpha}}^{2}\right)\left\|w^{1}\right\|_{H^{2}}^{2} .
$$

These inequalities, and $w^{1}$ and $w^{2}$ belonging to $H^{2}$, give (102).

Proposition 5. Let $\epsilon>0$ and $\alpha>0$ be such that $\epsilon+\alpha \leq 1$. There exists a real C depending only on $\lambda, \mu, w^{1}, w^{2}$, $e_{\Gamma}^{1}, e_{\Gamma}^{2}$, and $b$, such that

$$
\left\|N_{n}\left(e_{\Gamma}^{2}, w^{2}\right)-N_{n}\left(e_{\Gamma}^{1}, w^{1}\right)\right\|_{H^{-2}}^{2} \leq C \ln \left(1+\lambda_{n}\right)\|\tilde{w}\|_{H^{1}}^{2}+C \ln \left(1+\lambda_{n}\right)\left\|\tilde{e}_{\Gamma}\right\|_{L^{2}}^{2}+\frac{C}{\lambda_{n}^{\alpha}} .
$$


Proof. Let us define

$$
\begin{aligned}
X^{1}\left(\tilde{w}, w^{1}, w^{2}\right)= & -2 \lambda H\left\langle\nabla_{\Gamma} \tilde{w}, \nabla_{\Gamma}\left(w^{2}+w^{1}\right)\right\rangle+4 \lambda \operatorname{div}_{\Gamma}\left(H w^{2} \nabla_{\Gamma} \tilde{w}+H \tilde{w} \nabla_{\Gamma} w^{1}\right) \\
& +4 \mu \operatorname{div}_{\Gamma}\left(D^{2} b\left(w^{2} \nabla_{\Gamma} \tilde{w}+\tilde{w} \nabla_{\Gamma} w^{1}\right)\right) \\
& -2 \mu\left\langle D^{2} b \nabla_{\Gamma} \tilde{w}, \nabla_{\Gamma} w^{2}\right\rangle-2 \mu\left\langle D^{2} b \nabla_{\Gamma} w^{1}, \nabla_{\Gamma} \tilde{w}\right\rangle \\
& +(\lambda+2 \mu) \operatorname{div}_{\Gamma}\left(\nabla_{\Gamma} \tilde{w}\left\|\nabla_{\Gamma} w^{2}\right\|^{2}+\nabla_{\Gamma} w^{1}\left\langle\nabla_{\Gamma} \tilde{w}, \nabla_{\Gamma}\left(w^{2}+w^{1}\right)\right\rangle\right) \\
X^{2}\left(\tilde{w}, \tilde{e}_{\Gamma}, w^{1}, e_{\Gamma}^{1}, w^{2}, e_{\Gamma}^{2}\right)= & -2 \lambda \operatorname{div}_{\Gamma}\left(\operatorname{div}_{\Gamma} \tilde{e}_{\Gamma} \nabla_{\Gamma} w^{2}+\operatorname{div}_{\Gamma} e_{\Gamma}^{1} \nabla_{\Gamma} \tilde{w}\right) \\
& -4 \mu \operatorname{div}_{\Gamma}\left(\left(\varepsilon_{\Gamma} e_{\Gamma}^{2}+V_{\Gamma} e_{\Gamma}^{2}\right) \nabla_{\Gamma} \tilde{w}+\left(\varepsilon_{\Gamma} \tilde{e}_{\Gamma}+V_{\Gamma} \tilde{e}_{\Gamma}\right) \nabla_{\Gamma} w^{1}\right)
\end{aligned}
$$

so we have

$$
N_{n}\left(e_{\Gamma}^{2}, w^{2}\right)-N_{n}\left(e_{\Gamma}^{1}, w^{1}\right)=X^{1}\left(\tilde{w}, w^{1}, w^{2}\right)+X^{2}\left(\tilde{w}, \tilde{e}_{\Gamma}, w^{1}, e_{\Gamma}^{1}, w^{2}, e_{\Gamma}^{2}\right)
$$

and therefore

$$
\left\|N_{n}\left(e_{\Gamma}^{2}, w^{2}\right)-N_{n}\left(e_{\Gamma}^{1}, w^{1}\right)\right\|_{H^{-2}} \leq\left\|X^{1}\left(\tilde{w}, w^{1}, w^{2}\right)\right\|_{H^{-2}}+\left\|X^{2}\left(\tilde{w}, \tilde{e}_{\Gamma}, w^{1}, e_{\Gamma}^{1}, w^{2}, e_{\Gamma}^{2}\right)\right\|_{H^{-2}} .
$$

Estimation of the first term of the right hand side of (110)

$$
\begin{aligned}
\left\|X^{1}\left(\tilde{w}, w^{1}, w^{2}\right)\right\|_{H^{-2}} \leq & C\left(\left\|\left\langle\nabla_{\Gamma} \tilde{w}, \nabla_{\Gamma}\left(w^{2}+w^{1}\right)\right\rangle\right\|_{H^{-2}}+\left\|w^{2} \nabla_{\Gamma} \tilde{w}\right\|_{H^{-1}}\right. \\
& +\left\|\tilde{w} \nabla_{\Gamma} w^{1}\right\|_{H^{-1}}+\left\|\left\langle D^{2} b \nabla_{\Gamma} \tilde{w}, \nabla_{\Gamma} w^{2}\right\rangle\right\|_{H^{-2}}+\left\|\left\langle D^{2} b \nabla_{\Gamma} w^{1}, \nabla_{\Gamma} \tilde{w}\right\rangle\right\|_{H^{-2}} \\
& \left.+\left\|\operatorname{div}_{\Gamma}\left(\left(\varepsilon_{\Gamma} e_{\Gamma}^{2}+V_{\Gamma} e_{\Gamma}^{2}\right) \nabla_{\Gamma} \tilde{w}+\left(\varepsilon_{\Gamma} \tilde{e}_{\Gamma}+V_{\Gamma} \tilde{e}_{\Gamma}\right) \nabla_{\Gamma} w^{1}\right)\right\|_{H^{-2}}\right) .
\end{aligned}
$$

Bounding $\left\|X^{1}\left(\tilde{w}, w^{1}, w^{2}\right)\right\|_{H^{-2}}$ is an easy task, because all the terms are of lower order. For instance,

$$
\begin{aligned}
\left\|\left\langle\nabla_{\Gamma} \tilde{w}, \nabla_{\Gamma}\left(w^{2}+w^{1}\right)\right\rangle\right\|_{H^{-2}} & \leq C\left\|\left\langle\nabla_{\Gamma} \tilde{w}, \nabla_{\Gamma}\left(w^{2}+w^{1}\right)\right\rangle\right\|_{L^{2}} \\
& \leq C\left\|\nabla_{\Gamma} \tilde{w}\right\|_{L^{2}}\left\|\nabla_{\Gamma}\left(w^{2}+w^{1}\right)\right\|_{L^{2}} \\
& \leq C\|\tilde{w}\|_{H^{1}}\left\|w^{2}+w^{1}\right\|_{H^{1}} \\
& \leq C\|\tilde{w}\|_{H^{1}}
\end{aligned}
$$

where $C$ depends only on $w^{1}$ and $w^{2}$. An analogous argument is repeated for the four other terms. Eventually, we obtain that

$$
\left\|X^{1}\left(\tilde{w}, w^{1}, w^{2}\right)\right\|_{H^{-2}} \leq C\|\tilde{w}\|_{H^{1}}
$$

where $C$ depends only on $w^{1}, w^{2}$, the Lamé coefficients and $b$.

Estimation of the second term of the right hand side of (110)

$$
\begin{aligned}
\left\|X^{2}\left(\tilde{w}, \tilde{e}_{\Gamma}, w^{1}, e_{\Gamma}^{1}, w^{2}, e_{\Gamma}^{2}\right)\right\|_{H^{-2}} \leq & C\left(\left\|\operatorname{div}_{\Gamma}\left(\operatorname{div}_{\Gamma} \tilde{e}_{\Gamma} \nabla_{\Gamma} w^{2}+\operatorname{div}_{\Gamma} e_{\Gamma}^{1} \nabla_{\Gamma} \tilde{w}\right)\right\|_{H^{-2}}\right. \\
& \left.+\left\|\operatorname{div}_{\Gamma}\left(\left(\varepsilon_{\Gamma} e_{\Gamma}^{2}+V_{\Gamma} e_{\Gamma}^{2}\right) \nabla_{\Gamma} \tilde{w}+\left(\varepsilon_{\Gamma} \tilde{e}_{\Gamma}+V_{\Gamma} \tilde{e}_{\Gamma}\right) \nabla_{\Gamma} w^{1}\right)\right\|_{H^{-2}}\right) \\
\leq & C\left(\left\|\operatorname{div}_{\Gamma} \tilde{e}_{\Gamma} \nabla_{\Gamma} w^{2}\right\|_{H^{-1}}+\left\|\operatorname{div}_{\Gamma} e_{\Gamma}^{1} \nabla_{\Gamma} \tilde{w}\right\|_{H^{-1}}\right. \\
& \left.+\left\|\left(\varepsilon_{\Gamma} e_{\Gamma}^{2}+V_{\Gamma} e_{\Gamma}^{2}\right) \nabla_{\Gamma} \tilde{w}\right\|_{H^{-1}}+\left\|\left(\left(\varepsilon_{\Gamma} \tilde{e}_{\Gamma}+V_{\Gamma} \tilde{e}_{\Gamma}\right) \nabla_{\Gamma} w^{1}\right)\right\|_{H^{-1}}\right) .
\end{aligned}
$$

Let us look first at the term $\left\|\operatorname{div}_{\Gamma} \tilde{e}_{\Gamma} \nabla_{\Gamma} w^{2}\right\|_{H^{-1}}$. Using Green's formula for tangential derivatives (5) gives

$$
\begin{aligned}
\left\|\operatorname{div}_{\Gamma} \tilde{e}_{\Gamma} \nabla_{\Gamma} w^{2}\right\|_{H^{-1}}= & \sup _{\left\{\varphi,\|\varphi\|_{H_{0}^{1}}=1\right\}} \int_{\Gamma}\left(\operatorname{div}_{\Gamma} \tilde{e}_{\Gamma} \nabla_{\Gamma} w^{2}, \varphi\right)_{H^{-1} \times H_{0}^{1}} \\
= & \sup _{\left\{\varphi,\|\varphi\|_{H_{0}^{1}}=1\right\}} \int_{\Gamma}-\left\langle\tilde{e}_{\Gamma}, \nabla_{\Gamma}\left(\nabla_{\Gamma} w^{2}, \varphi\right)_{H^{-1} \times H_{0}^{1}}\right\rangle \\
& +2 \int_{\Gamma} H\left(\nabla_{\Gamma} w^{2}, \varphi\right)_{H^{-1} \times H_{0}^{1}}\left\langle\tilde{e}_{\Gamma}, \nabla b\right\rangle+\int_{\partial \Gamma}\left\langle\nabla_{\Gamma}\left(\nabla_{\Gamma} w^{2}, \varphi\right)_{H^{-1} \times H_{0}^{1}} \tilde{e_{\Gamma}}, \nu\right\rangle \\
= & -\sup _{\left\{\varphi,\|\varphi\|_{H_{0}^{1}}=1\right\}} \int_{\Gamma}\left\langle\tilde{e}_{\Gamma}, D_{\Gamma}^{2} w^{2} \varphi\right\rangle+\left\langle\tilde{e}_{\Gamma}, \nabla_{\Gamma} w^{2} D_{\Gamma} \varphi\right\rangle
\end{aligned}
$$


since the shell is clamped on the boundary and $\left\langle\tilde{e}_{\Gamma}, \nabla b\right\rangle=0$. Using Cauchy-Schwarz and the inequality (56) we have

$$
\begin{aligned}
\left|\int_{\Gamma}\left\langle\tilde{e}_{\Gamma}, D_{\Gamma}^{2} w^{2} \varphi\right\rangle\right| & =\left|\int_{\Gamma} D_{\Gamma}^{2} w^{2} \cdots\left(\tilde{e}_{\Gamma} \otimes \varphi\right)\right| \\
& \leq\left\|D_{\Gamma}^{2} w^{2}\right\|_{L_{2}}\left\|\tilde{e}_{\Gamma} \otimes \varphi\right\|_{L_{2}} \\
& \leq C\left|w^{2}\right|_{H^{2}}\left[\ln \left(1+\lambda_{n}\right)\left\|\tilde{e}_{\Gamma}\right\|_{L_{2}}+\frac{1}{\lambda_{n}^{\alpha}}\left\|\tilde{e}_{\Gamma}\right\|_{H^{\epsilon+\alpha}}\right]\|\varphi\|_{H^{1}}
\end{aligned}
$$

and the second term can be bounded as well:

$$
\begin{aligned}
\left|\int_{\Gamma}\left\langle\tilde{e}_{\Gamma}, \nabla_{\Gamma} w^{2} D_{\Gamma} \varphi\right\rangle\right| & =\left|\int_{\Gamma} D_{\Gamma} \varphi \cdots \tilde{e}_{\Gamma} \otimes \nabla_{\Gamma} w^{2}\right| \\
& \leq\left\|D_{\Gamma} \varphi\right\|_{L_{2}}\left\|\tilde{e}_{\Gamma} \otimes \nabla_{\Gamma} w^{2}\right\|_{L_{2}} \\
& \leq C\|\varphi\|_{H^{1}}\left[\ln \left(1+\lambda_{n}\right)\left\|\tilde{e}_{\Gamma}\right\|_{L_{2}}+\frac{1}{\lambda_{n}^{\alpha}}\left\|\tilde{e}_{\Gamma}\right\|_{H^{\epsilon+\alpha}}\right]\left\|w^{2}\right\|_{H^{2}}
\end{aligned}
$$

so that

$$
\left\|\operatorname{div}_{\Gamma} \tilde{e}_{\Gamma} \nabla_{\Gamma} w^{2}\right\|_{H^{-1}} \leq C\left[\ln \left(1+\lambda_{n}\right)\left\|\tilde{e}_{\Gamma}\right\|_{L_{2}}+\frac{1}{\lambda_{n}^{\alpha}}\left\|\tilde{e}_{\Gamma}\right\|_{H^{\epsilon+\alpha}}\right]\left\|w^{2}\right\|_{H^{2}} .
$$

The second and third terms are more direct

$$
\begin{aligned}
\left\|\operatorname{div}_{\Gamma} e_{\Gamma}^{1} \nabla_{\Gamma} \tilde{w}\right\|_{H^{-1}} & =\sup _{\left\{\varphi,\|\varphi\|_{H_{0}^{1}}=1\right\}} \int_{\Gamma} \operatorname{div}_{\Gamma} e_{\Gamma}^{1}\left\langle\nabla_{\Gamma} \tilde{w}, \varphi\right\rangle \\
& \leq \sup _{\left\{\varphi,\|\varphi\|_{H_{0}^{1}}=1\right\}}\left\|\operatorname{div}_{\Gamma} e_{\Gamma}^{1}\right\|_{L_{2}}\left\|\left\langle\nabla_{\Gamma} \tilde{w}, \varphi\right\rangle\right\|_{L_{2}} \\
& \leq C\left[\ln \left(1+\lambda_{n}\right)\|\tilde{w}\|_{H^{1}}+\frac{1}{\lambda_{n}^{\alpha}}\|\tilde{w}\|_{H^{1+\epsilon+\alpha}}\right]\left\|e_{\Gamma}^{1}\right\|_{H^{1}}
\end{aligned}
$$

and

$$
\begin{aligned}
\left\|\left(\varepsilon_{\Gamma}\left(e_{\Gamma}^{2}\right)+V_{\Gamma} e_{\Gamma}^{2}\right) \nabla_{\Gamma} \tilde{w}\right\|_{H^{-1}} & =\sup _{\left\{\varphi,\|\varphi\|_{H_{0}^{1}}=1\right\}} \int_{\Gamma}\left(\varepsilon_{\Gamma}\left(e_{\Gamma}^{2}\right)+V_{\Gamma} e_{\Gamma}^{2}\right) \cdots\left(\nabla_{\Gamma} \tilde{w} \otimes \varphi\right) \\
& \leq \sup _{\left\{\varphi,\|\varphi\|_{H_{0}^{1}}=1\right\}}\left\|\left(\varepsilon_{\Gamma}\left(e_{\Gamma}^{2}\right)+V_{\Gamma} e_{\Gamma}^{2}\right)\right\| L_{2}\left\|\nabla_{\Gamma} \tilde{w} \otimes \varphi\right\|_{L_{2}} \\
& \leq C\left[\ln \left(1+\lambda_{n}\right)\|\tilde{w}\|_{H^{1}}+\frac{1}{\lambda_{n}^{\alpha}}\|\tilde{w}\|_{H^{1+\epsilon+\alpha}}\right]\left\|e_{\Gamma}^{2}\right\|_{H^{1}} .
\end{aligned}
$$

The fourth term requires the following additional integrations using the Green's formulas (7):

$$
\begin{aligned}
\int_{\Gamma} \varepsilon_{\Gamma} \tilde{e}_{\Gamma} \cdots\left(\nabla_{\Gamma} w^{1} \otimes \varphi\right)= & \frac{1}{2} \int_{\Gamma} D_{\Gamma} \tilde{e}_{\Gamma} \cdots\left(\nabla_{\Gamma} w^{1} \otimes \varphi\right)+D_{\Gamma} \tilde{e}_{\Gamma} \cdots\left(\varphi \otimes \nabla_{\Gamma} w^{1}\right) \\
= & -\frac{1}{2} \int_{\Gamma}\left\langle\tilde{e}_{\Gamma}, \operatorname{div}_{\Gamma}\left(\nabla_{\Gamma} w^{1} \otimes \varphi\right)\right\rangle+\frac{1}{2} \int_{\Gamma} 2 H\left\langle\tilde{e}_{\Gamma},\left(\nabla_{\Gamma} w^{1} \otimes \varphi\right) \nabla b\right\rangle \\
& -\frac{1}{2} \int_{\Gamma}\left\langle\tilde{e}_{\Gamma}, \operatorname{div}_{\Gamma}\left(\varphi \otimes \nabla_{\Gamma} w^{1}\right)\right\rangle+\frac{1}{2} \int_{\Gamma} 2 H\left\langle\tilde{e}_{\Gamma},\left(\varphi \otimes \nabla_{\Gamma} w^{1}\right) \nabla b\right\rangle \\
= & -\frac{1}{2} \int_{\Gamma}\left\langle\tilde{e}_{\Gamma}, \operatorname{div}_{\Gamma}\left(\nabla_{\Gamma} w^{1} \otimes \varphi\right)+\operatorname{div}_{\Gamma}\left(\varphi \otimes \nabla_{\Gamma} w^{1}\right)\right\rangle+\int_{\Gamma} H\left\langle\tilde{e}_{\Gamma}, \nabla_{\Gamma} w^{1}\right\rangle\langle\varphi, \nabla b\rangle
\end{aligned}
$$

and

$$
V_{\Gamma} \tilde{e}_{\Gamma} \cdots\left(\nabla_{\Gamma} w^{1} \otimes \varphi\right)=\frac{1}{2}\left\langle D^{2} b \tilde{e}_{\Gamma}, \nabla_{\Gamma} w^{1}\right\rangle\langle\varphi, \nabla b\rangle
$$


so that we have

$$
\begin{aligned}
\left|\int_{\Gamma}\left(\varepsilon_{\Gamma}\left(\tilde{e}_{\Gamma}\right)+V_{\Gamma}\left(\tilde{e}_{\Gamma}\right)\right) \cdots\left(\nabla_{\Gamma} w^{1} \otimes \varphi\right)\right| \leq & \frac{1}{2}\left|\int_{\Gamma}\left\langle e_{\Gamma}, \operatorname{div}_{\Gamma}\left(\nabla_{\Gamma} w \otimes \varphi\right)+\operatorname{div}_{\Gamma}\left(\varphi \otimes \nabla_{\Gamma} w\right)\right\rangle\right| \\
& +\frac{1}{2}\left|\left\langle 2 H \tilde{e}_{\Gamma}+D^{2} b \tilde{e}_{\Gamma}, \nabla_{\Gamma} w^{1}\right\rangle\langle\varphi, \nabla b\rangle\right| \\
\leq & \frac{1}{2} \int_{\Gamma} \mid\left\langle\tilde{e}_{\Gamma}, D_{\Gamma}^{2} w^{1} \varphi\right\rangle+\left\langle\tilde{e}_{\Gamma}, \nabla_{\Gamma} w^{1} \operatorname{div}_{\Gamma} \varphi\right\rangle \\
& +\left\langle\tilde{e}_{\Gamma}, D_{\Gamma} \varphi \nabla_{\Gamma} w^{1}\right\rangle+\left\langle\tilde{e}_{\Gamma}, \Delta_{\Gamma} w^{1} \varphi\right\rangle \mid \\
& +C \int_{\Gamma}\left\langle\tilde{e}_{\Gamma}, \nabla_{\Gamma} w^{1}\right\rangle\langle\varphi, \nabla b\rangle \\
\leq & C\left(\left\|D_{\Gamma}^{2} w^{1}\right\|_{L_{2}}\left\|\tilde{e}_{\Gamma} \otimes \varphi\right\|_{L_{2}}+\left\|\operatorname{div}_{\Gamma} \varphi\right\|_{L_{2}}\left\|\left\langle\tilde{e}_{\Gamma}, \nabla_{\Gamma} w^{1}\right\rangle\right\|_{L_{2}}\right. \\
& +\left\|D_{\Gamma} \varphi\right\|_{L_{2}}\left\|\tilde{e}_{\Gamma} \otimes \nabla_{\Gamma} w^{1}\right\|_{L_{2}}+\left\|\Delta_{\Gamma} w^{1}\right\|_{L_{2}}\left\|\left\langle\tilde{e}_{\Gamma}, \varphi\right\rangle\right\|_{L_{2}} \\
& \left.+\left\|\left\langle\tilde{e}_{\Gamma}, \nabla_{\Gamma} w^{1}\right\rangle\right\|_{L_{2}}\|\langle\varphi, \nabla b\rangle\|_{L_{2}}\right)
\end{aligned}
$$

and after application of Eqs. (55) and (56) we have

$$
\left\|\left(\varepsilon_{\Gamma} \tilde{e}_{\Gamma}\right)+\left(V_{\Gamma} \tilde{e}_{\Gamma}\right) \nabla_{\Gamma} w^{1}\right\|_{H^{-1}} \leq C\left[\ln \left(1+\lambda_{n}\right)\left\|\tilde{e}_{\Gamma}\right\|_{L_{2}}+\frac{1}{\lambda_{n}^{\alpha}}\left\|\tilde{e}_{\Gamma}\right\|_{H^{\epsilon+\alpha}}\right]\left\|w^{1}\right\|_{H^{2}} .
$$

Finally, collecting equations (112) through (115) allows us to estimate the second term as

$$
\begin{aligned}
\left\|X^{2}\left(\tilde{w}, \tilde{e}_{\Gamma}, w^{1}, e_{\Gamma}^{1}, w^{2}, e_{\Gamma}^{2}\right)\right\|_{H^{-2}} \leq & C \ln \left(1+\lambda_{n}\right)\|\tilde{w}\|_{H^{1}}+C \ln \left(1+\lambda_{n}\right)\left\|\tilde{e}_{\Gamma}\right\|_{L_{2}} \\
& +\frac{C}{\lambda_{n}^{\alpha}}\|\tilde{w}\|_{H^{1+\epsilon+\alpha}}+\frac{C}{\lambda_{n}^{\alpha}}\left\|\tilde{e}_{\Gamma}\right\|_{H^{\epsilon+\alpha}}
\end{aligned}
$$

where $C$ depends only on $w^{1}, w^{2}$, the Lamé coefficients and $b$.

Conclusion: From (110), (111) and (116), we derive

$$
\left\|N_{n}\left(e_{\Gamma}^{2}, w^{2}\right)-N_{\Gamma}\left(e_{\Gamma}^{1}, w^{1}\right)\right\|_{H^{-2}}^{2} \leq C \ln \left(1+\lambda_{n}\right)\|\tilde{w}\|_{H^{1}}^{2}+C \ln \left(1+\lambda_{n}\right)\left\|\tilde{e}_{\Gamma}\right\|_{L^{2}}^{2}+\frac{C}{\lambda_{n}^{\alpha}}
$$

which is the announced result.

\subsection{Third step}

From now on we will consider $\alpha=1-\epsilon$. This choice of $\alpha$ complies with the requirements of Proposition 4 and Proposition 5.

Lemma 10. The following inequality holds true

$$
\left\|\tilde{e}_{\Gamma}\right\|_{L^{2}}^{2}+\|\tilde{w}\|_{H^{1}}^{2} \leq C t^{2} \lambda_{n}^{C t^{2}-\alpha}
$$

where $C$ depends only on $\mathbf{e}^{1}, \mathbf{e}^{2}$, the Lamé coefficients and the geometric information embedded in $b$.

Proof. Propositions 3-5 give

$$
\left\|\tilde{e}_{\Gamma}\right\|_{L^{2}}^{2}+\|\tilde{w}\|_{H^{1}}^{2} \leq t \int_{0}^{t}\left(C \ln \left(1+\lambda_{n}\right)\|\tilde{w}\|_{H^{1}}^{2}+C \ln \left(1+\lambda_{n}\right)\left\|\tilde{e}_{\Gamma}\right\|_{L^{2}}^{2}+\frac{C}{\lambda_{n}^{\alpha}}\right) \mathrm{d} s
$$

and therefore

$$
\left\|\tilde{e}_{\Gamma}\right\|_{L^{2}}^{2}+\|\tilde{w}\|_{H^{1}}^{2} \leq \frac{C t^{2}}{\lambda_{n}^{\alpha}}+C t \ln \left(1+\lambda_{n}\right) \int_{0}^{t}\left(\|\tilde{w}\|_{H^{1}}^{2}+\left\|\tilde{e}_{\Gamma}\right\|_{L^{2}}^{2}\right) \mathrm{d} s .
$$


Gronwall's inequality yields

$$
\left\|\tilde{e}_{\Gamma}\right\|_{L^{2}}^{2}+\|\tilde{w}\|_{H^{1}}^{2} \leq \frac{C t^{2}}{\lambda_{n}^{\alpha}} \exp \left(C t^{2} \ln \left(1+\lambda_{n}\right)\right)
$$

and consequently

$$
\left\|\tilde{e}_{\Gamma}\right\|_{L^{2}}^{2}+\|\tilde{w}\|_{H^{1}}^{2} \leq \frac{C t^{2}}{\lambda_{n}^{\alpha}}\left(1+\lambda_{n}\right)^{C t^{2}}
$$

Eq. (117) follows.

Corollary 3. We have $\tilde{\mathbf{e}}=0$.

Proof. The sequence $\left(\lambda_{n}\right)$ tends toward $+\infty$. When $t \in\left[0, \sqrt{\frac{\alpha}{C}}[\right.$, the right hand side of (117) tends to 0 . Consequently $\tilde{\mathbf{e}}=0$ on $\left[0, \sqrt{\frac{\alpha}{C}}[\right.$. The bootstrap argument completes the proof of the corollary and the proof of Theorem 2.

Remark 4. The proof of uniqueness does not give the continuous dependence, which requires a separate argument. This is the point of the next section of this paper.

\section{Proof of Theorem 3 (continuous dependence with respect to the initial data)}

The main technical difficulty in the proof of Theorem 3 is the derivation of the energy identity for weak solutions. We denote as $B([0, \tau[; X)$ the space of $X$-valued functions which are bounded on the interval $[0, \tau[$. This space is endowed with the norm $|x|_{B([0, \tau[; X)}=\sup _{t \in[0, \tau[}|x(t)|_{X}$.

\subsection{Energy identity result}

Lemma 11. We consider solutions to (16) with the following a priori regularity:

$$
\mathbf{e} \in B\left(\left[0, \tau\left[; V^{1}(\Gamma)\right), \quad \partial_{t} \mathbf{e} \in B\left(\left[0, \tau\left[; V^{0}(\Gamma)\right) .\right.\right.\right.\right.
$$

Then, the following energy identity holds:

$$
\mathcal{E}(t)=\mathcal{E}(s), \quad 0 \leq s \leq t \leq \tau
$$

where $\mathcal{E}$ is defined in (44).

Note that Proposition 1 cannot be applied as is because of the lack of regularity of e. In order to prove the energy identity we shall use a finite difference approximation of time derivatives (the same approximation as was used in [1, 18] for the von Kármán plate).

Let $h>0$ be a small parameter designed to go to zero. Let $g \in B([0, \tau[; X)$ where $X$ is a Hilbert space. We extend $g(t)$ to all $t \in \mathbb{R}$ by defining $g(t)=g(0)$ for $t \leq 0$ and $g(t)=g(\tau)$ for $t \geq \tau$. With these extensions we define three finite difference operators depending on the parameter $h$.

$$
\begin{aligned}
g_{h}^{+}(t) & \equiv g(t+h)-g(t) \\
g_{h}^{-}(t) & \equiv g(t)-g(t-h) \\
\partial_{h} g(t) & \equiv \frac{1}{2 h}\left[g_{h}^{+}(t)+g_{h}^{-}(t)\right] .
\end{aligned}
$$

Proposition 6. The following properties hold true:

(i) Let $g$ be weakly continuous with values in X. Then

$$
\lim _{h \rightarrow 0} \int_{0}^{\tau}\left(g(t), \partial_{h} g(t)\right)_{X} \mathrm{~d} t=\frac{1}{2}\left[\|g(\tau)\|_{X}^{2}-\|g(0)\|_{X}^{2}\right] .
$$


(ii) Let $g \in H^{1}\left(\left[0, \tau[; X)\right.\right.$. Then the following limits are well defined in $L_{2}([0, \tau[; X)$.

$$
\lim _{h \rightarrow 0} \partial_{h} g=\partial_{t} g ; \quad \lim _{h \rightarrow 0} \frac{1}{h} g_{h}^{+}=\partial_{t} g ; \quad \lim _{h \rightarrow 0} \frac{1}{h} g_{h}^{-}=\partial_{t} g .
$$

Moreover if $\partial_{t} g$ is weakly continuous with the values in $X$, then for every $t \in(0, \tau), \partial_{h} g(t) \rightarrow \partial_{t} g(t)$, weakly in $X$, and

$$
\frac{1}{h} g_{h}^{-}(\tau) \rightarrow \partial_{t} g(\tau) ; \quad \frac{1}{h} g_{h}^{+}(0) \rightarrow \partial_{t} g(0) ; \quad \text { weakly in } X .
$$

(iii) In addition to previous assumptions, let $V \subset X \subset V^{\prime}$,

$$
\partial_{t t} g \in L_{2}\left(\left[0, \tau\left[, V^{\prime}\right) ; \quad g \in L_{2}([0, \tau[, V),\right.\right.
$$

then

$$
\lim _{h \rightarrow 0} \int_{0}^{\tau}\left(\partial_{t t} g(t), \partial_{h} g(t)\right)_{X} \mathrm{~d} t=\frac{1}{2}\left[\left\|\partial_{t} g(\tau)\right\|_{X}^{2}-\left\|\partial_{t} g(0)\right\|_{X}^{2}\right] .
$$

Proof. The proof of Proposition 6 is elementary and the main steps are detailed in [18].

We return to the proof of Lemma 11.

Proof of Lemma 11. Eq. (47) gives

$$
\mathcal{E}^{F}(t) \equiv \mathcal{E}(t)-\frac{l}{2} \int_{\Gamma}\left(\mathcal{C} B_{M} \mathbf{e}, B_{M} \mathbf{e}\right)
$$

Recall that the definition of $\mathcal{E}^{F}$ includes the kinetic energy of the shell as well as the flexural terms from the potential energy $\mathcal{E}_{p}$ (see (46) and (35) for a definition of these quantities).

$$
\begin{aligned}
\mathcal{E}^{F}(t)= & \frac{\rho l}{2} \int_{\Gamma}\left|\partial_{t} e_{\Gamma}\right|^{2}+\left|\partial_{t} w\right|^{2}+\frac{\rho l \gamma}{2} \int_{\Gamma}\left|D^{2} b \partial_{t} e_{\Gamma}\right|^{2}+\left|\nabla_{\Gamma} \partial_{t} w\right|^{2}+\left|D^{2} b \partial_{t} e_{\Gamma}-\nabla_{\Gamma} \partial_{t} w\right|^{2} \\
& +\frac{\lambda \gamma l}{2}\left\langle\Delta_{\Gamma} w, \Delta_{\Gamma} \hat{w}\right\rangle_{\Gamma}+\mu \gamma l \int_{\Gamma} \operatorname{tr}\left(\left(S_{\Gamma} w+G_{\Gamma} w\right)\left(S_{\Gamma} \hat{w}+G_{\Gamma} \hat{w}\right)\right) .
\end{aligned}
$$

We will use the variational form (16) with the test functions $\partial_{h} \mathbf{e}=\partial_{h} \hat{e}_{\Gamma}+\partial_{h} \hat{w} \nabla b \in V^{1}(\Gamma)$. For reasons of readability we omit the details of the calculation, but this choice of test functions, dividing by 2 , and the first three formulas of Proposition 6 easily give the following identity:

$$
\frac{1}{2} \mathcal{E}^{F}(\tau)+\lim _{h \rightarrow 0} \int_{0}^{\tau} X_{h} \mathrm{~d} t=\frac{1}{2} \mathcal{E}^{F}(0)
$$

where, in terms of the tensor $B_{M}$, we have

$$
X_{h} \equiv \int_{\Gamma} \mathcal{C} B_{M}(\mathbf{e}) \cdots\left(\varepsilon\left(\partial_{h} e_{\Gamma}\right)+V_{\Gamma}\left(\partial_{h} e_{\Gamma}\right)+D^{2} b \partial_{h} w+\nabla_{\Gamma} w \otimes \nabla_{\Gamma} \partial_{h} w\right) .
$$

We note that

$$
\int_{\Gamma} \mathcal{C} B_{M} \mathbf{e} \cdots \partial_{h} B_{M} \mathbf{e}=\int_{\Gamma} \mathcal{C} B_{M}(\mathbf{e}) \cdots\left(\varepsilon\left(\partial_{h} e_{\Gamma}\right)+V_{\Gamma}\left(\partial_{h} e_{\Gamma}\right)+D^{2} b \partial_{h} w+\nabla_{\Gamma} w \otimes \nabla_{\Gamma} \partial_{h} w\right)
$$

so that we can rewrite $X_{h}$ as

$$
X_{h}=\int_{\Gamma} \mathcal{C} B_{M} \mathbf{e} \cdots \partial_{h} B_{M} \mathbf{e}-\int_{\Gamma} \mathcal{C} B_{M} \mathbf{e} \cdots\left(\frac{1}{2} \partial_{h}\left(\nabla_{\Gamma} w \otimes \nabla_{\Gamma} w\right)-\nabla_{\Gamma} w \otimes \nabla_{\Gamma} \partial_{h} w\right) .
$$

Direct calculations give us that

$$
\begin{aligned}
\frac{1}{2} \partial_{h}\left(\nabla_{\Gamma} w(t) \otimes \nabla_{\Gamma} w(t)\right) & =\frac{1}{4 h}\left[\nabla_{\Gamma} w(t+h) \otimes \nabla_{\Gamma} w(t+h)-\nabla_{\Gamma} w(t-h) \otimes \nabla_{\Gamma}(t-h)\right] \\
& =\frac{1}{2}\left[\partial_{h} \nabla_{\Gamma} w(t) \otimes \nabla_{\Gamma} w(t+h)+\nabla_{\Gamma} w(t-h) \otimes \partial_{h} \nabla_{\Gamma} w(t)\right] .
\end{aligned}
$$


Since $\mathcal{C}\left(B_{M} \mathbf{e}\right)$ is a symmetric tensor we have

$$
\begin{aligned}
\mathcal{C} & B_{M}(\mathbf{e}) \cdots\left(\frac{1}{2} \partial_{h}\left(\nabla_{\Gamma} w(t) \otimes \nabla_{\Gamma} w(t)\right)-\nabla_{\Gamma} w \otimes \nabla_{\Gamma} \partial_{h} w(t)\right) \\
= & \frac{1}{2} \mathcal{C} B_{M} \mathbf{e} \cdots\left(\partial_{h} \nabla_{\Gamma} w(t) \otimes \nabla_{\Gamma} w(t+h)-\nabla_{\Gamma} w \otimes \nabla_{\Gamma} \partial_{h} w(t)\right) \\
& +\frac{1}{2} \mathcal{C} B_{M} \mathbf{e} \cdots\left(\nabla_{\Gamma} w(t-h) \otimes \partial_{h} \nabla_{\Gamma} w(t)-\nabla_{\Gamma} w \otimes \nabla_{\Gamma} \partial_{h} w(t)\right) \\
= & \frac{1}{2} \mathcal{C} B_{M} \mathbf{e} \cdots\left(\partial_{h} \nabla_{\Gamma} w(t) \otimes \nabla_{\Gamma}\left(w(t+h)-\nabla_{\Gamma} w(t)\right)\right) \\
& +\frac{1}{2} \mathcal{C} B_{M} \mathbf{e} \cdots\left(\partial_{h} \nabla_{\Gamma} w(t) \otimes \nabla_{\Gamma}\left(w(t-h)-\nabla_{\Gamma} w(t)\right)\right) \\
= & \frac{1}{2} \mathcal{C} B_{M} \mathbf{e} \cdots\left(\partial_{h} \nabla_{\Gamma} w(t) \otimes\left(\nabla_{\Gamma} w_{h}^{+}(t)-\nabla_{\Gamma} w_{h}^{-}(t)\right)\right) .
\end{aligned}
$$

From these equations we see that

$$
X_{h}=\int_{\Gamma} \mathcal{C} B_{M} \mathbf{e} \cdots \partial_{h} B_{M} \mathbf{e}-\frac{1}{2} \int_{\Gamma} \mathcal{C} B_{M} \mathbf{e} \cdots\left(\partial_{h} \nabla_{\Gamma} w(t) \otimes\left(\nabla_{\Gamma} w_{h}^{+}(t)-\nabla_{\Gamma} w_{h}^{-}(t)\right)\right)
$$

and applying the first identity of Proposition 6 together with weak continuity of $B_{M} \mathbf{e}$ we have

$$
\lim _{h \rightarrow 0} \int_{0}^{\tau} X_{h} \mathrm{~d} t=\frac{1}{2}\left\|\mathcal{C}^{1 / 2} B_{M} \mathbf{e}(\tau)\right\|_{L_{2}(\Gamma)}^{2}-\frac{1}{2}\left\|\mathcal{C}^{1 / 2} B_{M} \mathbf{e}(0)\right\|_{L_{2}(\Gamma)}^{2}-\frac{1}{2} \lim _{h \rightarrow 0} \int_{0}^{\tau} Y_{h} \mathrm{~d} t
$$

with

$$
Y_{h} \equiv \int_{\Gamma} \mathcal{C} B_{M} \mathbf{e} \cdots\left(\partial_{h} \nabla_{\Gamma} w(t) \otimes\left(\nabla_{\Gamma} w_{h}^{+}(t)-\nabla_{\Gamma} w_{h}^{-}(t)\right)\right) .
$$

Our goal is to show that

$$
\lim _{h \rightarrow 0} \int_{0}^{\tau} Y_{h}=0 .
$$

Once this is shown, the energy identity will follow from combining (126) and (131). Eqs. (131) and (133) give

$$
\int_{0}^{\tau} X_{h} \mathrm{~d} t \rightarrow \frac{1}{2}\left\|\mathcal{C}^{1 / 2} B_{M} \mathbf{e}(\tau)\right\|_{L_{2}(\Gamma)}^{2}-\frac{1}{2}\left\|\mathcal{C}^{1 / 2} B_{M} \mathbf{e}(0)\right\|_{L_{2}(\Gamma)}^{2}
$$

and thus from (126) we have

$$
\mathcal{E}^{F}(\tau)+\left\|\mathcal{C}^{1 / 2} B_{M} \mathbf{e}(\tau)\right\|_{L_{2}(\Gamma)}^{2}=\mathcal{E}^{F}(0)+\left\|\mathcal{C}^{1 / 2} B_{M} \mathbf{e}(0)\right\|_{L_{2}(\Gamma)}^{2}
$$

which gives the result of Lemma 11 for points $s=0, t=\tau$. Other points in the interval are treated the same way, due to the fact that the argument is local.

It remains only to show that the Eq. (133) holds. The a priori regularity of weak solutions gives that $B_{M} \mathbf{e} \in$ $B\left(\left[0, \tau\left[; L_{2}(\Gamma)\right)\right.\right.$ so that the assertion follows if

$$
\int_{0}^{\tau}\left\|\partial_{h} \nabla_{\Gamma} w \otimes\left[\nabla_{\Gamma} w_{h}^{+}-\nabla_{\Gamma} w_{h}^{-}\right]\right\|_{L_{2}(\Gamma)} \rightarrow 0 \quad \text { as } h \rightarrow 0 .
$$

Applying Cauchy-Schwarz lets us define

$$
Z_{h}=\int_{0}^{\tau} h\left\|\partial_{h} \nabla_{\Gamma} w\right\|_{L_{4}(\Gamma)}^{2}+h^{-1}\left(\left\|\nabla_{\Gamma} w_{h}^{+}\right\|_{L_{4}(\Gamma)}^{2}+\left\|\nabla_{\Gamma} w_{h}^{-}\right\|_{L_{4}(\Gamma)}^{2}\right) \mathrm{d} t .
$$

The fact that $Z_{h} \rightarrow 0$ as $h \rightarrow 0$ follows by a density argument after we show that

$$
Z_{h} \leq c\left(\|w\|_{L_{2}\left(0, \tau ; H^{2}(\Gamma)\right)}+\left\|\partial_{t} w\right\|_{L_{2}\left(0, \tau ; H^{1}(\Gamma)\right)}\right) .
$$


We apply Sobolev's inequality $\|g\|_{L_{4}(\Gamma)} \leq C\|g\|_{W^{1,1}(\Gamma)}$ which is known to be true for the tangential derivatives on the shell mid-surface [11]. Letting $g=\left\|\partial_{h} \nabla_{\Gamma} w\right\|^{2}$ gives

$$
\begin{aligned}
h\left\|\partial_{h} \nabla_{\Gamma} w\right\|_{L_{4}}^{2} & \leq C h\left\|\partial_{h} \nabla_{\Gamma} w\right\|_{W^{1,1}}^{2} \leq C h\left\|\nabla_{\Gamma}\left|\partial_{h} \nabla_{\Gamma} w\right|^{2}\right\|_{L_{1}}+C h\left\|\partial_{h} \nabla_{\Gamma} w\right\|_{L_{1}}^{2} \\
& \leq C h \int_{\Gamma}\left|\nabla_{\Gamma}\left\langle\partial_{h} \nabla_{\Gamma} w, \partial_{h} \nabla_{\Gamma} w\right\rangle\right|+C h \int_{\Gamma}\left\|\partial_{h} \nabla_{\Gamma} w\right\|^{2} \\
& \leq C h\left\|\partial_{h} \nabla_{\Gamma} w\right\|_{L_{2}(\Gamma)}\left\|\partial_{h} \nabla_{\Gamma} w\right\|_{H^{1}(\Gamma)}+C h \int_{\Gamma}\left\|\partial_{h} \nabla_{\Gamma} w\right\|^{2} .
\end{aligned}
$$

Using the fact that $h \partial_{h}$ is bounded then gives that

$$
h\left\|\partial_{h} \nabla_{\Gamma} w\right\|_{L_{4}}^{2} \leq 2 C\left\|\nabla_{\Gamma} w\right\|_{H^{1}(\Gamma)}\left\|\partial_{h} \nabla_{\Gamma} w\right\|_{L_{2}(\Gamma)} .
$$

The argument is similar for the other terms.

\subsection{Completion of the proof}

Let us define

$$
\mathbf{x}(t)=\left(\mathbf{e}, \partial_{t} \mathbf{e}\right)=\left(e_{\Gamma}(t), w(t), \partial_{t} e_{\Gamma}(t), \partial_{t} w(t)\right)
$$

where $\mathbf{e}=e_{\Gamma}(t)+w(t) \nabla b$ is a weak solution to the small finite deflections shell problem at the time $t$ due to some initial data $\mathbf{e}(0)$.

We start with initial data $\mathbf{x}(0) \in \mathcal{H}$ such that $\mathbf{x}^{n}(0) \rightarrow \mathbf{x}(0)$ in $\mathcal{H}$ as $n \rightarrow \infty$. Our aim is to prove that

$$
\mathbf{x}^{n}(0) \rightarrow \mathbf{x}(0) \quad \text { in } C([0, \tau[, \mathcal{H}) .
$$

We will follow the road map presented in [18].

The potential energy defined by (35) only differs from the potential energy used in [5] by lower order terms. As a consequence, it is topologically equivalent to $V^{1}(\Gamma)$. In particular, using Sobolev's embedding and Korn's inequality (both of which are valid for the tangential calculus [11]) we have the inequalities

$$
\begin{aligned}
\left\|e_{\Gamma}\right\|_{H^{1}(\Gamma)} & \leq C\left[\left\|B_{M} \mathbf{e}\right\|_{L_{2}(\Gamma)}+\left\|\nabla_{\Gamma} w\right\|_{L_{4}(\Gamma)}^{2}+\left\|e_{\Gamma}\right\|_{L_{2}(\Gamma)}\right] \\
& \leq C\left[\left\|B_{M} \mathbf{e}\right\|_{L_{2}(\Gamma)}+\|w\|_{H^{2}(\Gamma)}^{2}+\left\|e_{\Gamma}\right\|_{L_{2}(\Gamma)}\right] .
\end{aligned}
$$

By Lemma 11 and the inequality (141), we have

$$
\|\mathbf{x}\|_{\mathcal{H}} \leq C \mathcal{E}(\mathbf{x}(t))
$$

so that

$$
\left\|\mathbf{e}^{n}(t)\right\|_{\mathcal{H}} \leq C\left(\left\|\mathbf{e}^{n}(0)\right\|_{\mathcal{H}}\right) \leq C\left(\|\mathbf{e}(0)\|_{\mathcal{H}}\right) .
$$

Hence, on a subsequence denoted by the same index we have

$$
\mathbf{x}^{n}(t) \rightarrow \mathbf{x}^{*}(t) \quad \text { weakly* in } L^{\infty}(0, \tau ; \mathcal{H}) .
$$

By using the variational equality together with weak continuity of nonlinear terms, we can show that $\mathbf{x}^{*}(t)$ coincides with a weak solution to (16) due to the initial data $\mathbf{x}(0)$. By uniqueness of the weak solution $\mathbf{x}^{*}(t)=\mathbf{x}(t)$. Hence we have

$$
\mathbf{x}^{n}(t) \rightarrow \mathbf{x}(t) \quad \text { weakly* in } L^{\infty}(0, \tau ; \mathcal{H}) .
$$

In view of this, to prove the theorem it is enough to show the norm convergence of

$$
\left\|\mathbf{x}^{n}(t)\right\|_{\mathcal{H}} \rightarrow\|\mathbf{x}(t)\|_{\mathcal{H}} \quad \text { in } C(0, \tau)
$$

We will use the equality in the energy relation from Lemma 11 to show this. We denote by $\mathcal{E}(\mathbf{x}(t))$ the energy corresponding to the solution $\mathbf{x}(t)$. From Lemma 11 we have

$$
\mathcal{E}\left(\mathbf{x}^{n}(t)\right)=\mathcal{E}\left(\mathbf{x}^{n}(0)\right) .
$$


By the continuity of energy with respect to the strong topology in $\mathcal{H}$ we have

$$
\lim _{n \rightarrow \infty} \mathcal{E}\left(\mathbf{x}^{n}(0)\right)=\mathcal{E}(\mathbf{x}(0))
$$

On the other hand by applying the lemma again we have

$$
\mathcal{E}(\mathbf{x}(0))=\mathcal{E}(\mathbf{x}(t))
$$

so that by uniqueness of weak solutions we have that

$$
\lim _{n \rightarrow \infty} \mathcal{E}\left(\mathbf{x}^{n}(t)\right)=\mathcal{E}(\mathbf{x}(t))
$$

where the limit is taken in $C(0, \tau)$. Combining weak convergence and norm convergence gives the convergence of the corresponding solutions in $C([0, \tau[; \mathcal{H})$.

\section{Acknowledgement}

The first and second authors' research was supported by NSF-INRIA grant number NSF-Int-0226961.

\section{References}

[1] A. Benabdallah, I. Lasiecka, Exponential decay rates for a full von Karman system of dynamic thermoelasticity, J. Differential Equations 160 (1) (2000) 51-93.

[2] M. Bernadou, Méthodes d'Eléments Finis pour les Problèmes de Coques Minces, Masson, Paris, 1994.

[3] M. Bernadou, J.T. Oden, An existence theorem for a class of nonlinear shallow shell problems, J. Math. Pures Appl. (9) 60 (3) (1981) $285-308$.

[4] A. Boutet de Montel, I. Chueshov, Uniqueness theorem for weak solutions of von Karman evolution equations, Comm. Partial Differential Equations 9 (1996) 267-294.

[5] J. Cagnol, I. Lasiecka, C. Lebiedzik, J.-P. Zolésio, Uniform stability in structural acoustic models with flexible curved walls, J. Differential Equations 186 (1) (2002) 88-121.

[6] J. Cagnol, C. Lebiedzik, On the free boundary conditions for a dynamic shell model based on intrinsic differential geometry, Appl. Anal. 83 (6) (2004) 607-633.

[7] I. Chueshov, Strong solutions and the attractors of the von Karman equations, Math. USSR Sbornik 69 (1991) $333-343$.

[8] P.G. Ciarlet, Un modèle bi-dimensionnel non linéaire de coque analogue à celui de W. T. Koiter, C. R. Acad. Sci. Paris Sér. I Math. 331 (5) (2000) 405-410.

[9] P.G. Ciarlet, P. Rabier, Les Equations de Von Karman, Springer-Verlag, 1982.

[10] P.G. Ciarlet, Theory of shells, in: Mathematical Elasticity vol. III, in: Studies in Mathematics and its Applications, vol. 29, North-Holland Publishing Co., Amsterdam, 2000.

[11] M. C. Delfour, J.-P. Zolésio, Intrinsic differential geometry and theory of thin shells (in press).

[12] M.C. Delfour, J.-P. Zolésio, Differential equations for linear shells: comparison between intrinsic and classical models, in: Advances in Mathematical Sciences, in: CRM Proc. Lect. Notes, vol. 11, AMS, 1997, pp. 41-124.

[13] M. Dikmen, Some recent advances in the dynamics of thin elastic shells. Linear theory, Internat. J. Eng. Sci. 17 (6) (1979) 659-680.

[14] G. Duvaut, J.-L. Lions, Les Inequations en Mecaniques et en Physiques, Dunod, 1972.

[15] A. Favini, I. Lasiecka, M.A. Horn, D. Tataru, Global existence, uniqueness and regularity of solutions to a von kármán system with nonlinear boundary dissipation, Differential Integral Equations 9 (2) (1996) 267-294.

[16] P. Germain, Mecanique, vol. 1, Ecole Polytechnique, 1986.

[17] L. Gratie, Two-dimensional nonlinear shell model of Koiter's type with variable thickness, Math. Mech. Solids 8 (6) (2003) $615-638$.

[18] H. Koch, I. Lasiecka, Hadamard well-posedness of weak solutions in nonlinear dynamic elasticity — full von Karman systems, Progr. Nonlinear Differential Equations Appl. 50 (2002) 197-216.

[19] H. Koch, A. Stachel, Global existence of classical solutions to the dynamical von Karman equations, Math. Methods Appl. Sci. 16 (1993) 581-586.

[20] W.T. Koiter, A consistent first approximation in the general theory of thin elastic shells, in: Proc. Sympos. Thin Elastic Shells (Delft, 1959), North-Holland, Amsterdam, 1960, pp. 12-33.

[21] W.T. Koiter, On the nonlinear theory of thin elastic shells. III, Nederl. Akad. Wetensch. Proc. Ser. B 69 (1966) $33-54$.

[22] J. Lagnese, Boundary Stabilization of Thin Plates, SIAM, Philadelphia, PA, 1989.

[23] J. Lagnese, G. Leugering, Uniform stabilization of a nonlinear beam by nonlinear boundary feedback, J. Differential Equations 91 (1991) 355-388.

[24] J. Lagnese, J.-L. Lions, Modelling Analysis and Control of Thin Plates, in: Research in Applied Mathematics, vol. 6, Masson, Paris, 1988.

[25] I. Lasiecka, Uniform stabilizability of a full von Karman system with nonlinear boundary feedback, SIAM J. Control. Optim. 36 (4) (1998) 1376-1422.

[26] I. Lasiecka, Weak, classical and intermediate solutions to full von Karman system of dynamic nonlinear elasticity, Appl. Anal. 68 (1998) $121-145$. 
[27] J.-L. Lions, E. Magenes, Problèmes aux Limites non Homogènes et Applications, Dunod, 1968.

[28] P.M. Naghdi, Foundations of elastic shell theory, in: Progress in Solid Mechanics, vol. IV, North-Holland, Amsterdam, 1963, pp. 1-90.

[29] J.L. Sanders Jr., Nonlinear theories for thin shells, Quart. Appl. Math. 21 (1963) 21-36.

[30] V.I. Sedenko, On uniqueness of the generalized solutions of initial boundary value problem for Marguerre-Vlasov nonlinear oscillations of the shallow shells, Rus. Izvestiya, North-Caucasus Region, Ser. Nat. Sciences 1-2 (1994).

[31] D. Tataru, M. Tucsnak, On the Cauchy problem for the full von Karman system, Nonlinear Differential Equations 4 (3) (1997) 325-340.

[32] T. von Kármán, Festigkeitprobleme in maschinenbau, Encyklopedie der Mathematischen Wissenschaften 4 (1910) 314-385. 